\title{
Vortex axisymmetrization: Dependence on azimuthal wave-number or asymmetric radial structure changes
}

\author{
By GERALD B. SMITH II and MICHAEL T. MONTGOMERY* \\ Colorado State University, USA
}

(Received 22 June 1994; revised 18 January 1995)

\section{SUMMARY}

Intense vortices in the atmosphere and ocean exhibit a high degree of axisymmetry despite persistent asymmetric forcing from their environment. To further elucidate vortex axisymmetrization a variety of idealized initial-value models for barotropic non-divergent flow is considered. To ensure basic understanding, disturbance evolution is first examined in a rectilinear system of simple shear. Particular emphasis is placed on identifying how inviscid disturbance-evolution depends on the zonal wave-number and on the meridional structure of the initial conditions.

Insight acquired from the rectilinear problem is then applied to a bounded Rankine vortex. Here, the dependency of disturbance evolution on the azimuthal wave-number is of special interest. Recent development of a low-frequency balance theory for rapidly rotating (large Rossby number) vortices has provided observational evidence that the low-azimuthal-wave-number asymmetries, especially wave number one, are dominant in the nearvortex region. The results of this work provide further theoretical evidence of an inviscid wave-number-selection mechanism that preferentially damps the high-wave-number asymmetries.

The radial structure and location of the initial conditions are found to be important factors in determining how rapidly a disturbance is compressed or elongated. This in turn controls the rate of disturbance growth or decay. For swirling flows, a definition of an effective shear is proposed that accounts for both the radial variations of the initial condition and the radial variation of the angular velocity. Using the reciprocal of this effective shear, time-scales for a disturbance to decay to half its initial energy, the half-life, are calculated for initial conditions and symmetric wind-profiles that are found in hurricanes.

Simple-shear flow and the bounded Rankine vortex do not admit discrete modal solutions since there is no mean-state vorticity-gradient to support them. The unbounded Rankine vortex is examined to investigate how the presence of discrete modes (Rossby edge-waves) associated with the radial vorticity-gradient of the Rankine swirl modifies the continuous spectrum solutions presented here.

KEYWORDS: Axisymmetry Hurricanes Rankine flow Vortices

\section{INTRODUCTION}

Intense vortices observed in the atmosphere and ocean exhibit a high degree of axisymmetry, despite persistent asymmetric forcing from their environment. In hurricanes, the interaction between the vortex and environmental asymmetries has implications for spiral-band dynamics (Willoughby 1977; Guinn and Schubert 1993), intensification (or weakening) processes (Molinari 1992), and storm motion (Shapiro and Ooyama 1990; Shapiro 1992; Smith and Weber 1993). Thus, understanding the asymmetric dynamics of intense vortices is critical for understanding the physical mechanisms controlling vortex evolution.

Until recently, the full primitive equations have been the favoured tool for investigating the three-dimensional asymmetric dynamics of intense vortices. Formulation of a lowfrequency balance theory, in which high-frequency gravity-and inertial-waves are filtered while retaining the pertinent aspects of advection, is complicated for rapidly rotating (large Rossby number) vortices since the time-scale of the tangential advection is comparable to that of the gravity- and inertial-waves on the vortex. Shapiro and Montgomery (1993, hereafter SM) proposed a three-dimensional asymmetric balance (AB) theory that includes the full inertial effects of the rapidly rotating region while filtering the gravity-and inertialwaves. In the vortex environment, $\mathrm{AB}$ theory reduces to quasi-geostrophic balance. In the absence of asymmetries, $\mathrm{AB}$ theory reduces to Eliassen's axisymmetric-balance model (Eliassen 1951) throughout the vortex.

* Corresponding author: Department of Atmospheric Science, Colorado State University, Fort Collins, CO 80523, USA. 
SM were able to separate the advective processes from the inertial and gravity waves by defining a local Rossby number that accounted for the varying rotation in an intense vortex. The local Rossby number is given as the ratio of the orbital frequency to the inertial frequency multiplied by the azimuthal wave-number (AWN). Based on observations from Hurricane Gloria in 1985, SM showed that the square of the local Rossby number is generally less than unity only for wave number one in the rapidly rotating region of an intense vortex. One of the goals of this work is to further elucidate the wave-number-selection mechanism that provides the basis for $\mathrm{AB}$ theory. To obtain a more comprehensive picture of the physics of axisymmetrization and its fundamental role in hurricane evolution, previous theoretical studies using quasi-geostrophic shallow-water models and nondivergent barotropic models are briefly reviewed.

Melander et al. (1987) examined vortex axisymmetrization in a quasi-geostrophic shallow-water model using a pseudo-spectral formulation. The model was initialized using finite-amplitude elliptical vortices with modified 'top hat' radial profiles. In that paper, axisymmetrization was identified with the shedding of vorticity filaments outside the nearly-axisymmetric-vortex core. Rather than decompose the results into radial and azimuthal components, vortex evolution was diagnosed by plotting the aspect ratio of vorticity contours as a function of time. At early times ( $t$ less than a few orbital periods), all aspect ratios decreased towards unity and then began small-amplitude oscillations. Melander $e t$ al. identified this initial transition with axisymmetrization.

For the majority of cases they considered, representation of dissipative processes at small scales was parametrized using a hyperviscosity formulation in the potential-vorticityevolution equation. In principle, non-zero diffusion could conceivably have played a role in the axisymmetrization process since diffusion is always scale-selective. However, by increasing the value of the hyperviscosity used in their model, Melander et al. convincingly demonstrated the inviscid nature of the axisymmetrization process.

In a complementary approach, McCalpin (1987) used a reduced-gravity quasi-geostrophic model to study axisymmetrization in Gulf Stream rings. The vortex model consisted of finite-amplitude azimuthal-mode- 2 or mode- 3 perturbations superposed on an axisymmetric Gaussian basic state. McCalpin found that nearly all the perturbation energy was transferred to the basic state on time-scales of the order of an orbital period. While only weakly dependent on the perturbation strength, the decay time-scales were found to be strongly dependent on the strength of the mean flow and the AWN. In particular, the decay time-scale for wave number three was found to be $60 \%$ faster than that of wave number two.

Like Melander et al., McCalpin parametrized dissipative processes with a hyperviscosity formulation. However, only a limited discussion regarding the effects of diffusion on the symmetrization process was provided. McCalpin calculated a diffusion time-scale that appears to be based on $L^{4} / A_{\mathrm{B}}$, where $L$ is the characteristic horizontal scale and $A_{\mathrm{B}}$ is the hyperviscosity coefficient. For deformation-radius-scale waves, his diffusion time-scale was found to be 80 years. However, since the differential rotation in the vortex rapidly reduces the radial scale of the asymmetries (SM; also Sutyrin 1989), one must not neglect this effect when estimating the diffusion time-scale.

In terms of McCalpin's model parameters, an estimate of the diffusion time-scale that incorporates the differential rotation is

$$
t_{d} \sim \frac{1}{A_{\mathrm{B}}\left[\left(4 \pi^{2} m^{2} n^{2}+n^{2}\right) / r^{2}\right]^{2}}
$$

where $m$ is the number of orbits traversed by a fluid parcel at the radius of maximum winds $(r)$ and $n$ is the AWN. For $A_{\mathrm{B}}=5 \times 10^{9} \mathrm{~m}^{4} \mathrm{~s}^{-1}, m=1, n=2$ and $r=50 \mathrm{~km}, t_{\mathrm{d}}$ is found 
to be approximately 13 hours. In calculating $t_{\mathrm{d}}$, it is assumed that the differential rotation has already decreased the radial scale of an asymmetry to render basic-state quantities effectively constant in a first approximation. Based on the results of Melander et al., this occurs in roughly a few orbital periods. For a typical run, McCalpin found the perturbationdecay time-scale to be 1.5 orbital periods. This suggests that viscous influences were probably insignificant.

Both McCalpin and Melander et al. simulated asymmetric-vortex evolution using fully non-linear numerical models that generally prevent analytical solutions. While their results represent important and meaningful contributions to our understanding of axisymmetrization, basic physical processes may be masked by the presence of non-linear dynamics, diffusion and the $\beta$-effect. Because of uncertainties in our knowledge of horizontal and vertical eddy-diffusivities in geophysical vortices, it is particularly important to understand the purely inviscid problem.

Sutyrin (1989) developed a formal solution for linear inviscid disturbance-evolution in a quasi-geostrophic shallow-water model on an $f$-plane. In this model, the disturbance potential-vorticity (PV) was conserved following fluid particles. For regions where the basic-state PV was identically zero, Sutyrin showed that the non-modal component of the disturbance PV became oscillatory in radius with the oscillations controlled by the differential rotation of the fluid. In addition, the radial gradient of the non-modal disturbance PV was shown to increase linearly with time. Sutyrin asserted that a similar non-modal disturbance-evolution would be observed in regions with a continuous and monotonic basic-state PV profile.

The linear increase with time of the non-modal disturbance PV gradient was fundamentally due to the reduction of the radial scale of the disturbance. Sutyrin concluded, then, that the symmetrization process was due to the differential rotation of the fluid and was analogous to perturbation decay in rectilinear flow with simple shear (Case 1960).

Like Melander et al., Sutyrin did not examine the possibility of an inviscid AWNselection mechanism nor did he investigate the energy transfer between the asymmetries and the circularly symmetric basic state. Identification of these processes is complicated in Sutyrin's model since the solution is expressed in terms of an operator on an infinite function-space.

Carr and Williams (1989, hereafter CW) examined vortex axisymmetrization using an inviscid non-divergent barotropic model. Their evolutionary model consisted of smallamplitude perturbations superposed on a steady axisymmetric Rankine flow. CW were primarily interested in the asymmetry-inducing influences of $\beta$ and environmental windshear on hurricane vortices.

$\mathrm{CW}$ suggested that the damping rate of perturbations is proportional to the square of the AWN. This conclusion was based on results derived from initial conditions in which both the AWN and the radial structure changed simultaneously. CW suggested further that their result was analogous to the dependence of damping on the zonal wave-number (ZWN) as described by Case for plane Couette flow. However, a careful review of Case's paper did not reveal any discussion of how the perturbation damping-rate and the ZWN are related. Since this relationship is potentially a fundamental aspect of an inviscid wavenumber-selection mechanism in sheared flows, such as hurricanes and other geophysical vortices, both Case and $\mathrm{CW}$ will be reconsidered to clarify this issue.

In this work, axisymmetrization will be studied as an initial-value problem within the framework of inviscid non-divergent vorticity dynamics. Extension to a non-divergent system would permit analyses of the effects of a finite (and variable) deformation-radius as well as the influence of gravity-wave emission on the axisymmetrization process. These interesting topics remain for future work. Since the curvature vorticity and the curvilinear 
coordinate-system of a swirling vortex may complicate understanding of the disturbance dynamics, the analogous rectilinear simple-shear model (Case 1960; Farrell 1987) will be examined first. Identification of how the disturbance evolution depends on the $\mathrm{ZWN}$ and the meridional structure of the initial condition will be emphasized.

\section{Algebraic EVOLUTION IN UNBOUNDED SIMPLE SHEAR}

In this section the evolution of small-amplitude perturbations in the presence of simple shear is examined as a first step towards understanding the evolution of asymmetries in a hurricane. Since observations suggest that the asymmetric winds in hurricanes are small compared to the symmetric tangential wind in the near-vortex region (SM), linear perturbation-theory is used to express the flow as a meridionally-varying basic-state and a small-amplitude perturbation field. Of particular interest is the dependence of the disturbance evolution on the ZWN and the meridional structure of an initial vorticity-profile. The flow is assumed to be unbounded and the model employed is based on the two-dimensional inviscid incompressible Euler equations on an $f$-plane. Since non-divergent dynamics is ultimately controlled by the vorticity equation, the Coriolis parameter will not directly affect the interpretation of results.

\section{(a) Model development}

In Cartesian coordinates the linearized vorticity-equation for an incompressible fluid is given by

$$
\frac{\partial \zeta}{\partial t}+\bar{u} \frac{\partial \zeta}{\partial x}+v \frac{\mathrm{d} \bar{\zeta}}{\mathrm{d} y}=0
$$

where

$$
\zeta=\frac{\partial v}{\partial x}-\frac{\partial u}{\partial y}
$$

is the vertical component of perturbation relative vorticity, $(x, y)$ are the zonal and meridional coordinates, $(u, v)$ are the zonal and meridional perturbation winds, and an overbar denotes basic-state quantities. For simple-shear flow, the basic-state wind is given by $\bar{u}=S y$ where $S$ is a constant shear. The vorticity equation (2.1) then becomes

$$
\frac{\partial \zeta}{\partial t}+S y \frac{\partial \zeta}{\partial x}=0
$$

which states that the perturbation vorticity is conserved following the basic-state flow. In an infinite domain, $u$ and $v$ must be bounded as $|x|$ or $|y|$ becomes arbitrarily large.

To satisfy continuity, a perturbation streamfunction is defined such that

$$
u=-\frac{\partial \psi}{\partial y} \quad v=\frac{\partial \psi}{\partial x} \text {. }
$$

Equation (2.3) is thus

$$
\left(\frac{\partial}{\partial t}+S y \frac{\partial}{\partial x}\right)\left(\frac{\partial^{2}}{\partial x^{2}}+\frac{\partial^{2}}{\partial y^{2}}\right) \psi=0
$$

Following the methodology of Case (1960), the Fourier-space solution $\hat{\psi}$ for the perturbation streamfunction is found to be

$$
\hat{\psi}(k, y, t)=\int_{-\infty}^{\infty} G\left(y, y_{0}\right) \hat{\zeta}_{0}\left(k, y_{0}\right) e^{-\mathrm{i} k S t y_{0}} \mathrm{~d} y_{0}
$$


where

$$
G\left(y, y_{0}\right)=-\frac{1}{2 k} \begin{cases}e^{k\left(y-y_{0}\right)}, & y \leqslant y_{0} \\ e^{-k\left(y-y_{0}\right)}, & y \geqslant y_{0}\end{cases}
$$

is the Green's function, $k$ is the ZWN, and $\hat{\zeta}_{0}(k, y)$ is the initial Fourier vorticity for wave number $k$. Equation (2.6) is the continuous-spectrum solution to (2.5) since it results from integrating over all advective frequencies $-\mathrm{i} k \bar{u}\left(y_{0}\right)$ spanned by the flow.

The solution is completed by applying the inverse Fourier transform, yielding

$$
\psi(x, y, t)=\frac{1}{2 \pi} \int_{-\infty}^{\infty} e^{\mathrm{i} k x} \int_{-\infty}^{\infty} G\left(y, y_{0}\right) \hat{\zeta}_{0}\left(k, y_{0}\right) e^{-\mathrm{i} k S y_{0} t} \mathrm{~d} y_{0} \mathrm{~d} k
$$

For profiles of initial vorticity $\hat{\zeta}_{0}=2 \pi \bar{\zeta}_{0}(y) \delta\left(k_{*}-k\right)$ representing a monochromatic disturbance of $\mathrm{ZWN}=k$ and meridional structure $\hat{f}$, the Fourier inversion gives

$$
\psi_{k}(x, y, t)=\int_{-\infty}^{\infty} G\left(y, y_{0}\right) \hat{\zeta}_{0}\left(y_{0}\right) e^{\mathrm{i} k\left(x-S y_{0} t\right)} \mathrm{d} y_{0} .
$$

For simplicity, $k$ is used to represent the ZWN in both physical and Fourier space. Since viscosity has been neglected in (2.9), all processes are time-reversible. The convention will be to define $t=0$ as the initial time, $t>0$ as future times, and $t<0$ as past times.

\section{(b) Plane wave reviewed}

A simple initial condition in an infinite domain is a plane-wave disturbance. Although many properties of this solution are well known (Thomson 1887; Orr 1907; Farrell 1982; Lindzen 1990), it is essential to review this particular case since it will be shown to play an important role in characterizing the limiting-decay behaviour of sheared disturbances. Let

$$
\hat{\zeta}_{0}=-\left(k^{2}+l_{0}^{2}\right) e^{i l_{0} y},
$$

where $l_{0}$ is the initial meridional wave-number (MWN). Substituting (2.10) into (2.9), integrating, and taking the real part yields

$$
\psi_{k}=\frac{k^{2}+l_{0}^{2}}{k^{2}+\left(l_{0}-k S t\right)^{2}} \cos \left\{k x+\left(l_{0}-k S t\right) y\right\}
$$

The quantity $l_{0}-k S t$ is a time-dependent $\mathrm{MWN}, l$, which describes the ever-changing amplitude and meridional structure of the perturbation. For $k>0$, the MWN and time are negatively related so as $t$ increases $l$ decreases.

As an aid to understanding the evolution of the physical fields, consider the wave vector $\mathbf{k}=(k, l)$. The magnitude of the wave vector $|\mathbf{k}|$, is the total wave-number of the perturbation. The angle the wave vector makes with the zonal axis (measured in a counterclockwise sense from the positive $x$ axis) is given by $\theta=\tan ^{-1}(l / k)$. The wave vector is perpendicular to lines of constant phase, so $\theta$ ranges from $-\pi / 2$ to $\pi / 2$.

The streamfunction evolution described by (2.11) is qualitatively considered for fixed $k$ and $l_{0}$. The maximum streamfunction amplitude is $1+l_{0}^{2} / k^{2}$ and occurs when $t=l_{0} / k S(l=0)$. Thus, the streamfunction amplitude grows when $t<l_{0} / k S(l>0)$ and it decays for $t>l_{0} / k S(l<0)$. When the streamfunction amplitude is maximum, the streamfunction field is parallel to the meridional axis and the number of waves in the domain is given by $k$. Since $t$ and $l$ are negatively related, increasing $t$ results in decreasing $\theta$, so the streamfunction field rotates clockwise. However, increasing (decreasing) $t$ results 
in $|\mathbf{k}|$ decreasing (increasing) when $t<l_{0} / k S\left(t>l_{0} / k S\right)$. When the streamfunction field is in the growth (decay) phase the number of waves in the domain decreases (increases). Comparing the slope of the streamfunction contours with the slope of the shear, the streamfunction field tilts against the shear in the growth phase while the streamfunction field tilts with the shear in the decay phase. When the streamfunction field is upright, its amplitude is maximum.

To further quantify the growth and decay, an expression for the perturbation kineticenergy (KE) density is derived. Substituting (2.11) into (2.4) gives the zonal and meridional winds for the plane-wave streamfunction. These are

$$
\begin{aligned}
& u_{k}=\frac{\left(l_{0}-k S t\right)\left(k^{2}+l_{0}^{2}\right)}{k^{2}+\left(l_{0}-k S t\right)^{2}} \sin \left\{k x+\left(l_{0}-k S t\right) y\right\}, \\
& v_{k}=-\frac{k\left(k^{2}+l_{0}^{2}\right)}{k^{2}+\left(l_{0}-k S t\right)^{2}} \sin \left\{k x+\left(l_{0}-k S t\right) y\right\} .
\end{aligned}
$$

The KE density is then $\rho\left(u^{2}+v^{2}\right) / 2$, which gives

$$
E_{k}=\frac{\left(k^{2}+l_{0}^{2}\right)^{2}}{2\left\{k^{2}+\left(l_{0}-k S t\right)^{2}\right\}} \sin ^{2}\left\{k x+\left(l_{0}-k S t\right) y\right\}
$$

where $\rho$ has been set to one.

Equation (2.13) gives a local measure of the KE density. A more useful measure which isolates the temporal evolution is an integrated KE. For the plane wave, the integration is performed over one wavelength zonally and meridionally since the integrated KE density over the entire plane is infinite. The integration yields

$$
\left\langle E_{k}\right\rangle=\frac{\left(k^{2}+l_{0}^{2}\right)^{2}}{4\left\{k^{2}+\left(l_{0}-k S t\right)^{2}\right\}}
$$

where $\left\langle E_{k}\right\rangle$ is the integrated KE per wave. This is maximum when $l_{0}-k S t=0$, which gives

$$
\left\langle E_{k}\right\rangle_{\max }=\frac{\left(k^{2}+l_{0}^{2}\right)^{2}}{4 k^{2}} .
$$

Thus, the normalized KE per wave (hereafter, the normalized KE) is

$$
\frac{\left\langle E_{k}\right\rangle}{\left\langle E_{k}\right\rangle_{\max }}=\frac{1}{1+\left(l_{0} / k-S t\right)^{2}} .
$$

The normalization has been done with the maximum value of the normalized KE rather than the initial value so that the normalized KE ranges from zero to one. From (2.16), the instantaneous growth (decay) rate is found to be

$$
\frac{1}{\left\langle E_{k}\right\rangle} \frac{\mathrm{d}\left\langle E_{k}\right\rangle}{\mathrm{d} t}=\frac{2 k S\left(l_{0}-k S t\right)}{k^{2}+\left(l_{0}-k S t\right)^{2}} .
$$

The maximum growth (decay) rate occurs when the time derivative of (2.17) vanishes. This corresponds to $S t=\mp 1+l_{0} / k, l= \pm k$ and $\theta= \pm \pi / 4$. At these times, the normalized KE is 0.5 .

This suggests a natural evolution time-scale for the half-life of the perturbation energy. For the plane-wave perturbation, the half-life is $1 / S$. Interestingly, this is independent of $k$ 

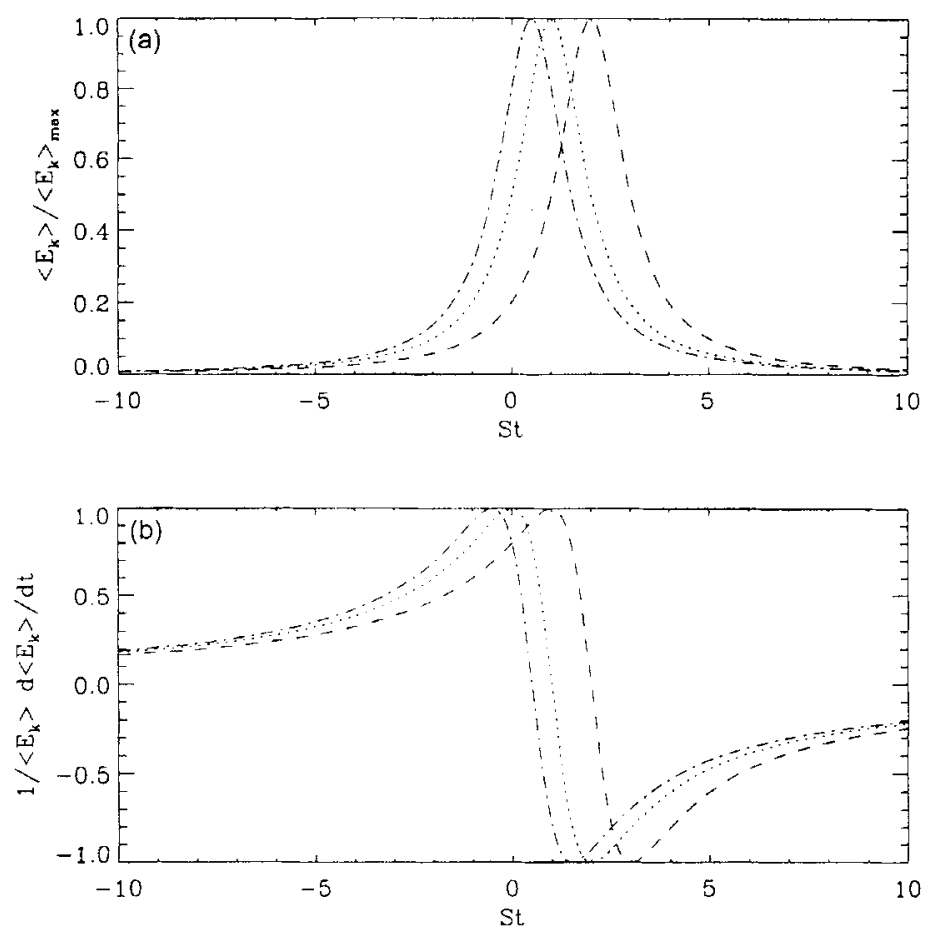

Figure 1. (a) Normalized kinetic energy and (b) growth (decay) rates for a plane-wave initial vorticity-profile with $k=1, l_{0}=1$ (dot); $k=1, l_{0}=2$ (dash); $k=2, l_{0}=1$ (dot dash).

and $l_{0}$. Figure 1 shows the normalized $\mathrm{KE}$ and the growth (decay) rate as functions of time for $k=1, l_{0}=1 ; k=1, l_{0}=2$; and $k=2, l_{0}=1$. This figure clearly demonstrates that changing $k$ and $l_{0}$ merely shifts the energy curve along the time axis, but does not change the structure of the energy curve.

The half-life for horizontally-sheared disturbances in mid-latitude cyclones, hurricanes and tornadoes may be estimated as follows. Letting $L$ denote a typical horizontal scale and $U$ a typical horizontal speed, a characteristic half-life time is given by $L / U$. In moderate mid-latitude cyclones, $U \approx 10 \mathrm{~m} \mathrm{~s}^{-1}$ and $L \approx 1000 \mathrm{~km}$ giving a horizontal shear of $10^{-5} \mathrm{~s}^{-1}$ and a half-life of about 1 day. In the eyewall of a hurricane, $U \approx 50 \mathrm{~m} \mathrm{~s}^{-1}$ and $L \approx 50 \mathrm{~km}$. Thus, the horizontal shear is $10^{-3} \mathrm{~s}^{-1}$ and the half-life about 15 minutes. Finally, in a tornado, $U \approx 100 \mathrm{~m} \mathrm{~s}^{-1}$ and $L \approx 100 \mathrm{~m}$ yielding a horizontal shear of $1 \mathrm{~s}^{-1}$ and a half-life of 1 second. While only approximate, these numbers simply illustrate how the shear strength affects the evolution time-scale.

Perturbation growth and decay imply an energy transfer to and from the disturbance. The instantaneous tendency of the perturbation energy is given by

$$
\frac{\partial\langle E\rangle}{\partial t}=-\iint u v \frac{\mathrm{d} \bar{u}}{\mathrm{~d} y} \mathrm{~d} x \mathrm{~d} y .
$$

From (2.12) and (2.18), it is clearly seen that for perturbation growth (decay) the net momentum flux is negative (positive). The instantaneous tendency of the shear, then, is to decrease (increase) and the basic state loses (gains) energy. Although the total KE is conserved in the non-linear case, perturbation $\mathrm{KE}$ is not conserved in the linear one since the basic state acts as an infinite reservoir.

While previous work has emphasized the algebraic growth-phase of disturbance evo- 
lution for a variety of important meteorological problems (e.g. Farrell 1982, 1984, 1987; Montgomery and Farrell 1990,1991), axisymmetrization is a decay process and attention is hereafter restricted to disturbances that are initially upright. The upright-plane-wave initial condition $\left(l_{0}=0\right)$ is formally equivalent to a constant initial vorticity-profile. Thus, apart from a shifting along the time axis, wave perturbations in simple shear forced by either constant or meridionally-periodic initial conditions have normalized KE (2.16) that is independent of the $\mathrm{ZWN} k$.

\section{(c) Gaussian initial vorticity}

The plane wave considered in the previous section provides analytical solutions for the perturbation evolution in simple shear. However, transient atmospheric forcings are more realistically represented as isolated disturbances. To introduce a meridional scale to the initial vorticity, a Gaussian profile is considered. Physically, this could correspond to a meridionally-confined short-wave trough in the mid-latitude jet. The Gaussian is given by

$$
\hat{\zeta}_{0}(y)=e^{-\alpha y^{2}}
$$

where $\alpha^{-1 / 2}$ defines characteristic $e$-folding length-scale. Unlike the plane-wave initial condition, when (2.19) is substituted into (2.9), closed-form solutions are not available.

Using the trapezoidal rule to evaluate the integral, Figs. 2 and 3 plot streamfunction and vorticity contours respectively, for the profile of initial vorticity $\hat{\zeta}_{0}=\exp \left(-y^{2}\right)$. The rows show ZWNs $k=1,2$ and 3 respectively, while the columns denote non-dimensional times $S t=0.0,1.0,2.0$ respectively. There are several differences between the Gaussian and the upright-plane-wave cases. For a fixed time, the streamfunction amplitude decreases with increasing $k$. In addition, the figure suggests that higher-wave-number perturbations decay faster than lower-wave-number perturbations. In the upright plane wave, the streamfunction amplitude and the decay rate were independent of the ZWN. Finally, there is a suggestion that for large time, the plane-wave solution emerges.

As with the plane-wave perturbation, a global measure of the KE is desired. Here, though, the meridional integration is from $-\infty$ to $+\infty$. Figure 4 shows the normalized $\mathrm{KE}$ as a function of time for the initial vorticity-profile $\hat{\zeta}_{0}=\exp \left(-y^{2}\right)$. As indicated in Fig. 4, higher-wave-number perturbations decay more rapidly than lower-wave-number perturbations. In addition, for increasing $k$ or $t$, the normalized KE curves approach the plane-wave solution asymptotically. Interestingly, the wave number $k=1$ perturbation decays significantly slower than all higher-wave-number perturbations. This would not be surprising in a viscous fluid. However, in the above results the viscosity is identically zero yet higher-wave-number disturbances still decay faster than smaller-wave-number disturbances. This suggests a truly inviscid decay mechanism that rapidly damps the higher wave-numbers leaving the lower wave-numbers behind.

To gain further understanding of the ZWN dependence in the evolution of meridionally-confined initial vorticity-disturbances, it is instructive to examine the interaction of the three terms comprising the integrand of (2.9). As $k$ increases, the Green's function narrows while the phase function oscillates more rapidly. The effect of increasing $k$ is for the product of the Green's function and the phase function to become more meridionally confined. For large enough $k$, the meridional scale of this product is so small that the initial vorticity appears effectively constant in the integration. This is reminiscent of the uprightplane-wave solution in which the initial vorticity-profile is constant in the integration. Thus, while the meridional scale of the initial vorticity generally introduces a dependence on the ZWN in the shear dynamics, for large $k$ the plane-wave solution should emerge. 

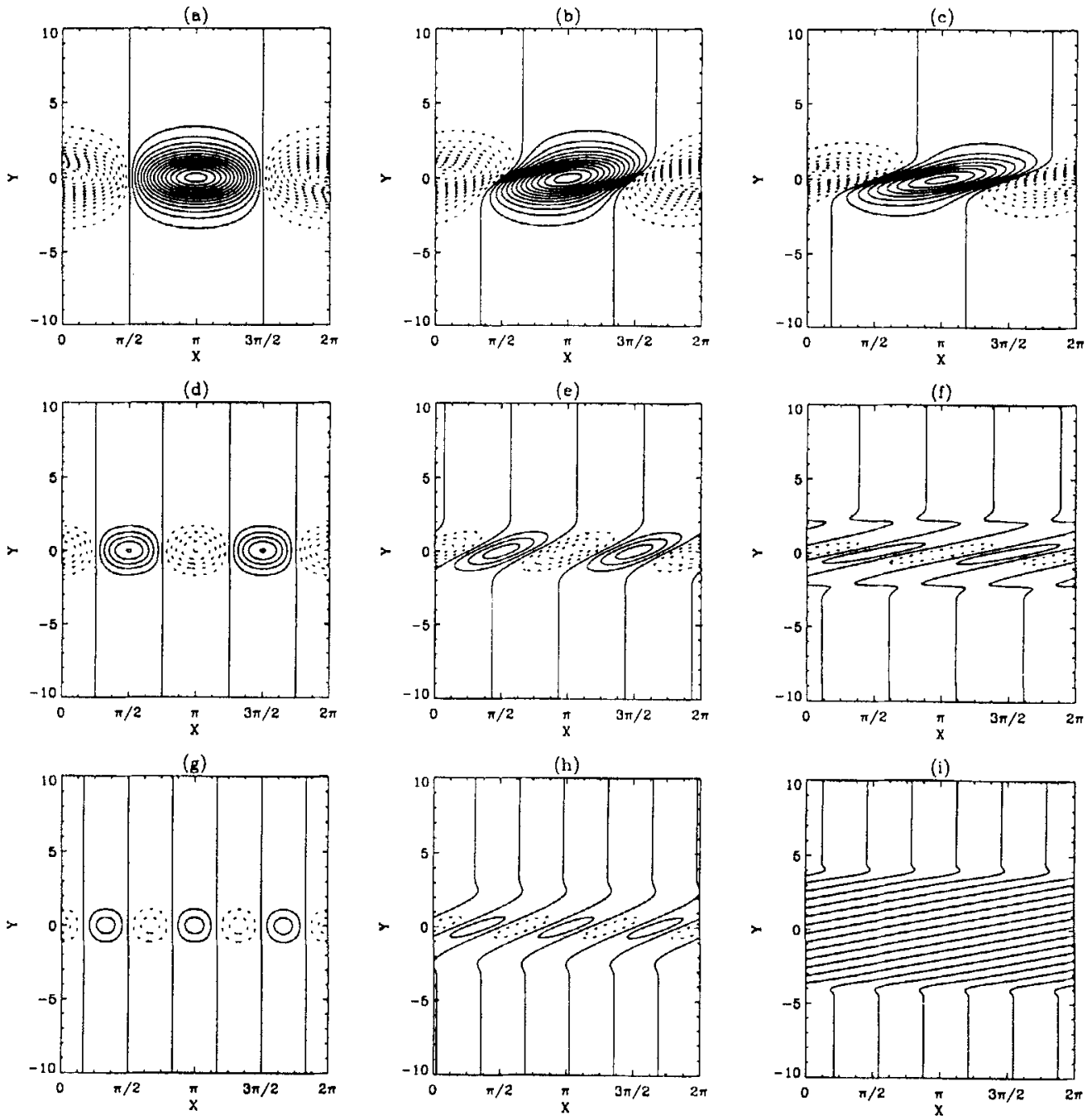

Figure 2. Perturbation streamfunction for the Gaussian initial vorticity-profile $\hat{\zeta}_{0}=\exp \left(-y^{2}\right)$. Panels (a)-(c) show contours for zonal wave-number $k=1$ at times $S t=0.0,1.0$ and 2.0 respectively. Panels (d) $-(\mathrm{f})$ show contours for $k=2$ and panels (g)-(i) show contours for $k=3$ at the same times. The contour interval is $3.76 \times 10^{-2} \mathrm{~m}^{2} \mathrm{~s}^{-1}$ in all panels. Solid contours denote $\psi_{k} \geqslant 0$, dotted contours $\psi_{k}<0$.

For increasing $k$, the plane-wave solution may be recovered from a meridionallyconfined initial condition as follows. The streamfunction amplitude in Fourier space is

$$
\hat{\psi}(y)=\int_{-\infty}^{\infty} G\left(y, y_{0}\right) \hat{\zeta}_{0}\left(y_{0}\right) e^{-i k S t y_{0}} \mathrm{~d} y_{0} .
$$

As $k$ becomes large, the streamfunction amplitude can be approximated by

$$
\hat{\psi}(y) \approx \hat{\zeta}_{0}(y) \int_{-\infty}^{\infty} G\left(y, y_{0}\right) e^{-i k S t y_{0}} \mathrm{~d} y_{0} \quad 1 \ll k<\infty .
$$

This integral may be evaluated exactly giving the approximate streamfunction-amplitude

$$
\hat{\psi}=-\frac{\hat{\zeta}_{0}(y)}{k^{2}\left\{1+(S t)^{2}\right\}} e^{-i k s t y}
$$




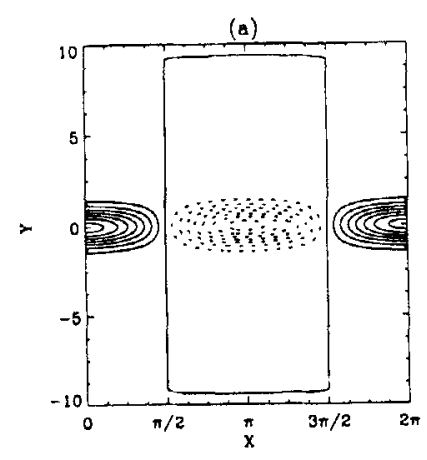

(d)
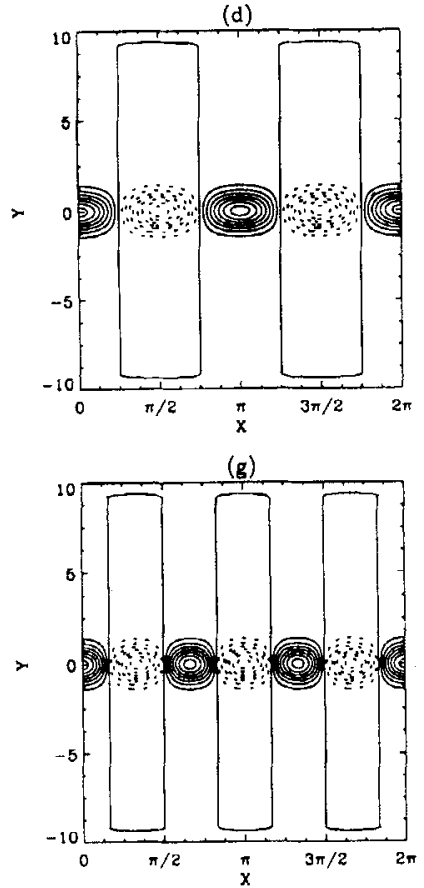

(b)

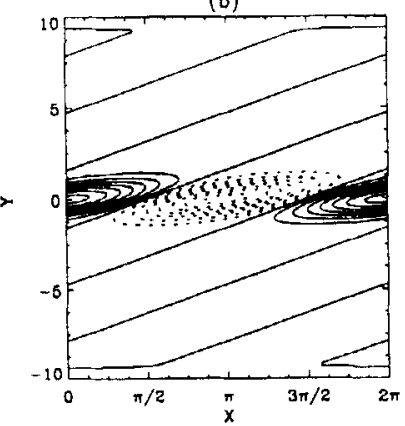

(e)

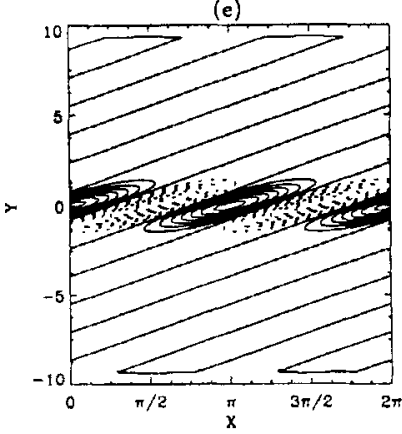

(h)

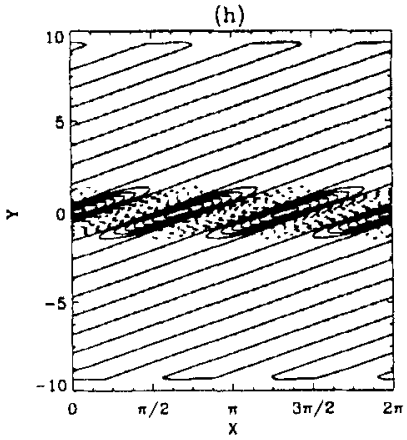

(c)

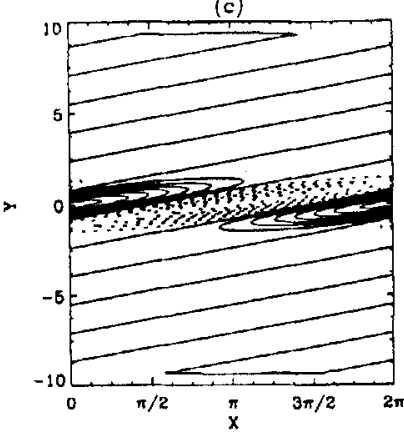

(t)

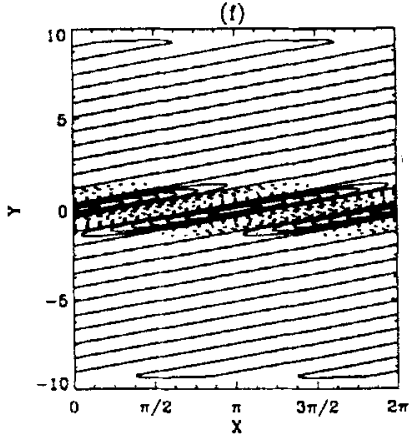

(i)

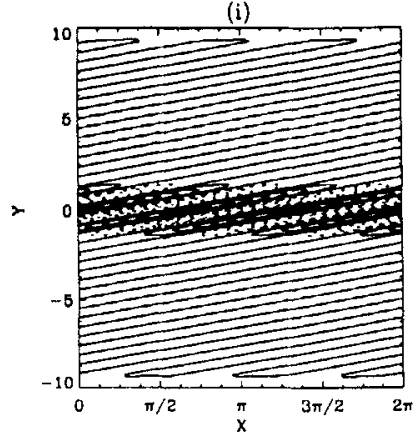

Figure 3. Perturbation vorticity for the Gaussian initial vorticity-profile $\hat{\zeta}_{0}=\exp \left(-y^{2}\right)$. The panels are the same as in Fig. 2. The contour interval is $1.33 \times 10^{-1} \mathrm{~s}^{-1}$ in all panels.

Upon applying the inverse Fourier transform and taking the real part, (2.22) becomes

$$
\psi_{k}=-\frac{\hat{\zeta}_{0}(y)}{k^{2}\left\{1+(S t)^{2}\right\}} \cos \{k(x-S t y)\}
$$

so the meridional velocity is

$$
v_{k}=\frac{\hat{\zeta}_{0}(y)}{k\left\{1+(S t)^{2}\right\}} \sin \{k(x-S t y)\}
$$

Now, the zonal velocity is

$$
u_{k}=-\frac{\mathrm{d} \hat{\zeta}_{0}(y)}{\mathrm{d} y} \frac{\cos \{k(x-S t y)\}}{k^{2}\left\{1+(S t)^{2}\right\}}-\frac{\hat{\zeta}_{0}(y) S t}{k\left\{1+(S t)^{2}\right\}} \sin \{k(x-S t y)\} .
$$




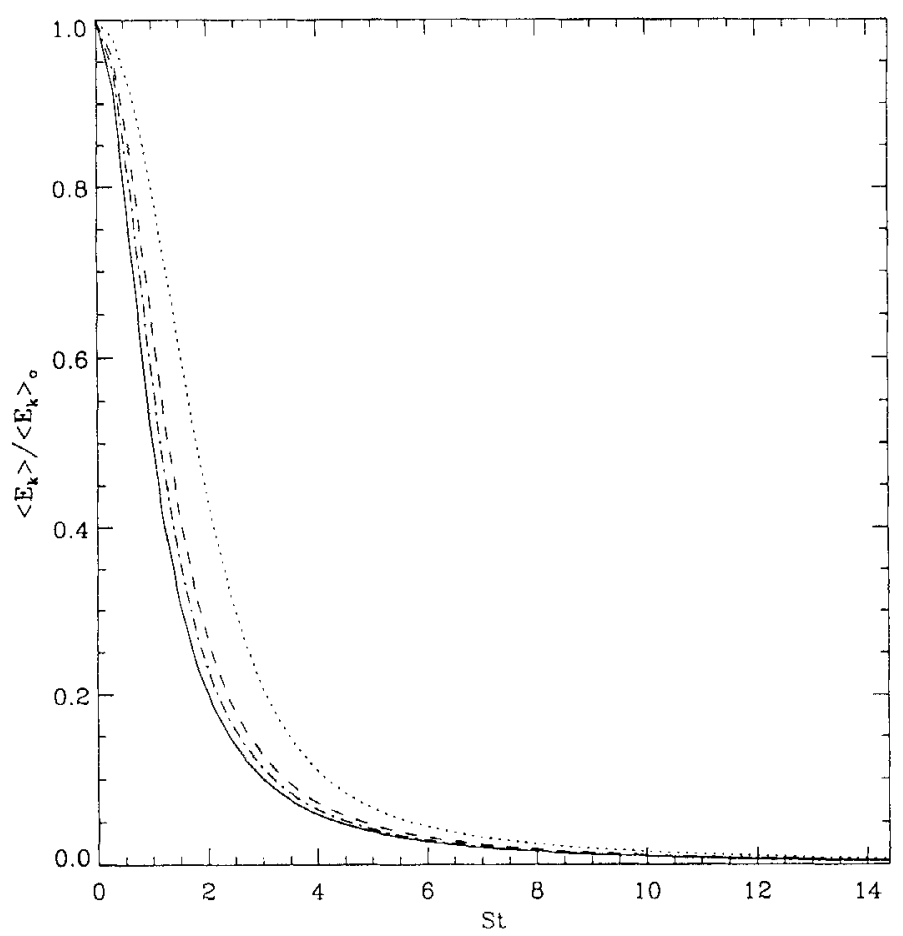

Figure 4. Normalized kinetic energy for the Gaussian initial condition $\hat{\zeta}_{0}=\exp \left(-y^{2}\right)$ for $k=1$ (dot), $k=2$ (dash) and $k=3$ (dot dash). The solid curve is the normalized kinetic energy for the upright plane wave.

Assuming $k$ large enough but finite such that the first term on the right side of (2.25) is small compared to the second term, the zonal velocity becomes

$$
u_{k} \approx-\frac{\hat{\zeta}_{0}(y) S t}{k\left\{1+(S t)^{2}\right\}} \sin \{k(x-S t y)\} .
$$

The KE density is then approximated by

$$
E_{k}=\frac{1}{2} \frac{\hat{\zeta}_{0}^{2}(y)}{k^{2}\left\{1+(S t)^{2}\right\}} \sin ^{2}\{k(x-S t y)\}
$$

where the density, $\rho$, has been set to one, while the integrated KE is given by

$$
\left\langle E_{k}\right\rangle=\frac{\pi}{2 k^{3}\left\{1+(S t)^{2}\right\}} \int_{-\infty}^{\infty} \hat{\zeta}_{0}^{2}(y) \mathrm{d} y .
$$

Normalizing (2.28) by its initial value

$$
\left\langle E_{k}\right\rangle_{0}=\frac{\pi}{2 k^{3}} \int_{-\infty}^{\infty} \hat{\zeta}_{0}^{2}(y) \mathrm{d} y,
$$

gives

$$
\frac{\left\langle E_{k}\right\rangle}{\left\langle E_{k}\right\rangle_{0}}=\frac{1}{1+(S t)^{2}}
$$


the normalized $\mathrm{KE}$ for the upright plane wave.

Figure 4 shows the plane-wave solution emerging for large time. As time increases, the phase function in (2.20) oscillates more rapidly while the Green's function and initial condition remain unchanged. For sufficiently large times, the half-wavelength scale of the phase function is narrow enough so that the initial condition appears locally constant which is analogous to the upright plane wave. Further, double integration by parts of (2.20) subject to the boundary conditions reveals the $t^{-2}$ dependence of the streamfunction amplitude at large times.

Figures 5 and 6 plot perturbation streamfunction and vorticity contours, respectively, for $\mathrm{ZWN} k=1$. The rows show the fields for initial vorticity-profiles $\hat{\zeta}_{0}=\exp \left(-\alpha y^{2}\right)$ where $\alpha=1,2$ and 3 respectively, while the columns denote the fields at non-dimensional times $S t=0.0,1.0$ and 2.0 respectively. Figure 7 plots the corresponding normalized $\mathrm{KE}$ as a function of time and shows that decreasing the meridional scale of the initial condition results in slower energy-decay. Since the energy decay is related to the half-wavelength scale of the phase function, decreasing the scale of the initial condition increases the time required for a given amount of energy decay. Thus, small-scale disturbances approach the plane-wave solution more slowly than large-scale disturbances.

Physically, disturbance evolution is governed by the conservation of disturbance vorticity so the meridional change in the zonal wind across a disturbance controls how rapidly a vorticity disturbance is elongated. Because the area of a fluid element does not change in non-divergent flow, an elongating vorticity-patch implies an attendant decrease in the disturbance meridional-scale. The decrease in the meridional scale is directly related to the decrease in disturbance energy. Disturbances of large meridional extent are elongated more rapidly than those of small meridional extent and, thus, decay more rapidly. Since the plane-wave solution spans the domain, its meridional length-scale is effectively infinite. Therefore, the plane wave always decays more rapidly than any meridionally-localized disturbance.

In a similar fashion, the dependence on the ZWN may be explained. For an initially upright disturbance, the ZWN is inversely related to the initial zonal extent of a disturbance. Now consider two disturbances with the same initial energy and meridional extent where one disturbance has twice the initial zonal extent of the other. In a given amount of time, the zonal elongation of both disturbances is the same. To conserve area, the change in the meridional scale of a disturbance with large zonal extent is less than that of a disturbance with small zonal extent. Therefore, a lower-wave-number disturbance decays more slowly than a higher-wave-number disturbance.

\section{AlgeBRAIC DECAY IN BOUNDED SIMPLE SHEAR}

Before examining axisymmetrization in the bounded Rankine vortex, the decay of perturbations in bounded simple shear is briefly examined.

\section{(a) Bounded rectilinear shear}

The presence of boundaries does not alter the governing equations used in section 2 . Nevertheless, meridional boundaries provide a convenient length-scale that may be used to non-dimensionalize the equations. Letting $(x, y)=L(\tilde{x}, \tilde{y}), \zeta=S \tilde{\zeta}$ and $t=\tilde{t} / S$, where $L$ is the boundary separation and the tildes denote non-dimensional quantities, the linearized vorticity-equation (2.3) in streamfunction notation becomes

$$
\left(\frac{\partial}{\partial \tilde{t}}+\tilde{y} \frac{\partial}{\partial \tilde{\lambda}}\right) \nabla^{2} \tilde{\psi}=0 .
$$


(a)

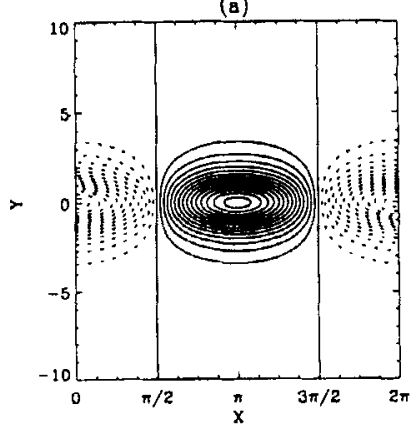

(d)

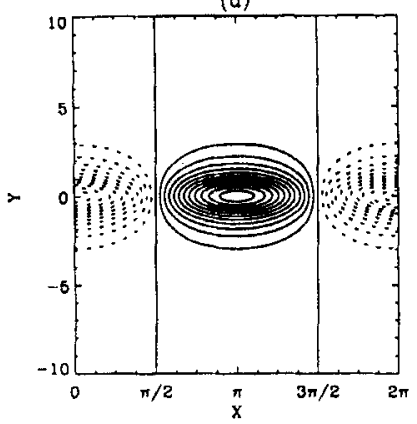

(g)

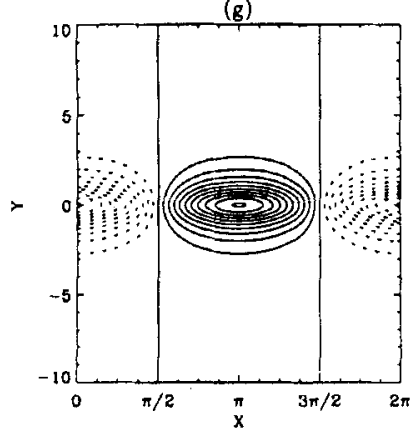

(b)

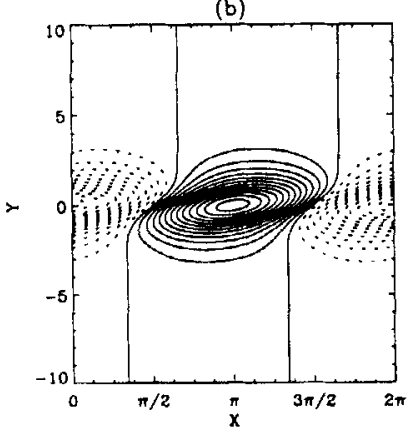

(e)

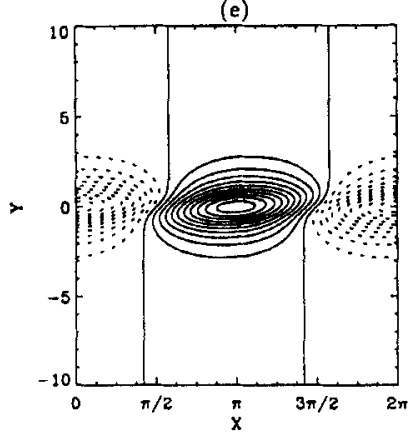

(h)

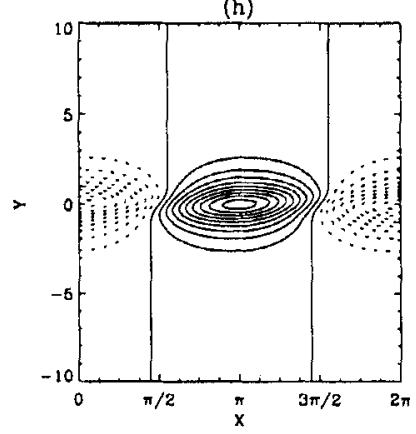

(c)

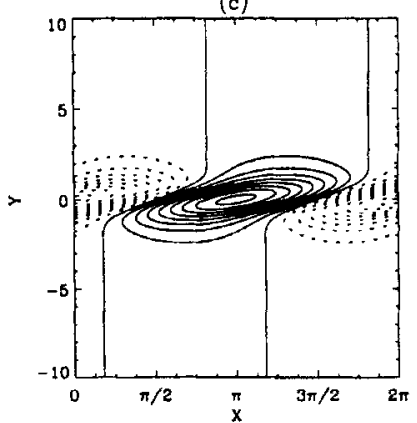

(I)

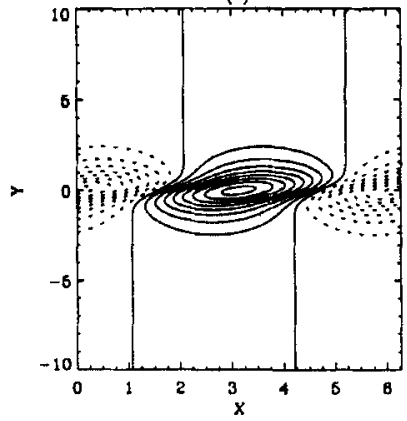

(i)

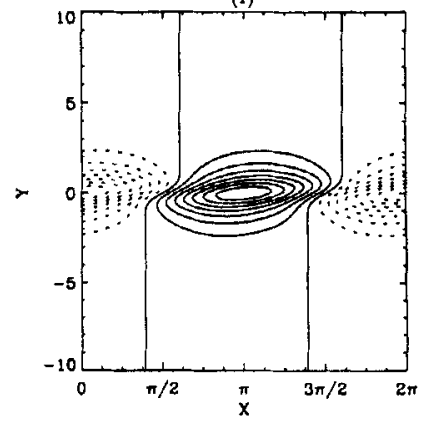

Figure 5. Perturbation streamfunction for zonal wave-number $k=1$. Panels (a)-(c) show contours for the initial vorticity-profile $\hat{\zeta}_{0}=\exp \left(-y^{2}\right)$ at times $S t=0.0,1.0$ and 2.0 respectively. Panels (d)-(f) show contours for the initial vorticity-profile $\zeta_{0}=\exp \left(-2 y^{2}\right)$ and panels (g)-(i) show contours for the initial vorticity-profile $\hat{\zeta}_{0}=$ $\exp \left(-3 y^{2}\right)$ at the same times. The contour interval is $3.76 \times 10^{-2} \mathrm{~m}^{2} \mathrm{~s}^{-1}$ in all panels. Solid contours are $\psi_{k} \geqslant 0$, dotted contours $\psi_{k}<0$.

Although the magnitude of the shear is no longer explicit in (3.1), the evolutionary time is scaled by the shear. Solutions to (3.1) depend on the choice of boundaries. Here, the meridional boundaries are assumed to be slippery walls at $y=a$ and $y=b$ where $a<b$. Thus, to satisfy the no-normal-flow condition $\tilde{v}(a)=\tilde{v}(b)=0$.

Again, following the methodology of Case, the Fourier-space solution to (3.1) is

$$
\hat{\psi}(k, y, t)=\int_{a}^{b} G\left(y, y_{0}\right) \hat{\zeta}_{0}\left(k, y_{0}\right) e^{-\mathrm{i} k t y_{0}} \mathrm{~d} y_{0}
$$

where

$$
G\left(y, y_{0}\right)=\frac{1}{k \sinh \{k(b-a)\}}\left\{\begin{array}{ll}
\sinh \{k(y-a)\} \sinh \left\{k\left(y_{0}-b\right)\right\} & a \leqslant y \leqslant y_{0} \\
\sinh \{k(y-b)\} \sinh \left\{k\left(y_{0}-a\right)\right\} & y_{0} \leqslant y \leqslant b
\end{array} .\right.
$$


(a)

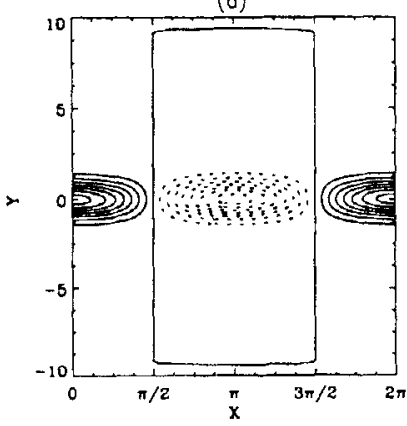

(d)

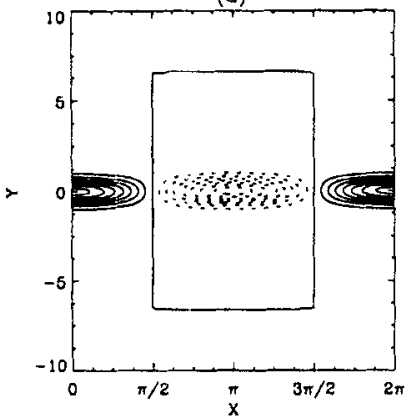

(g)

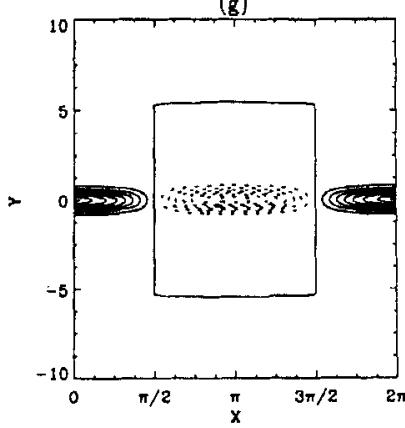

(b)

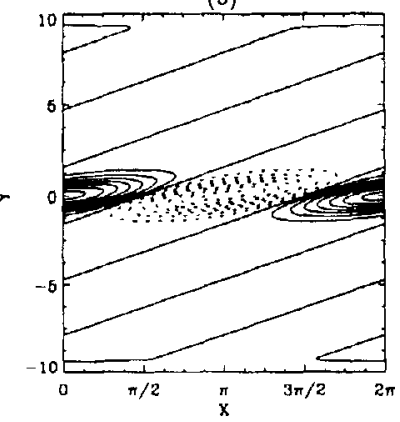

(e)

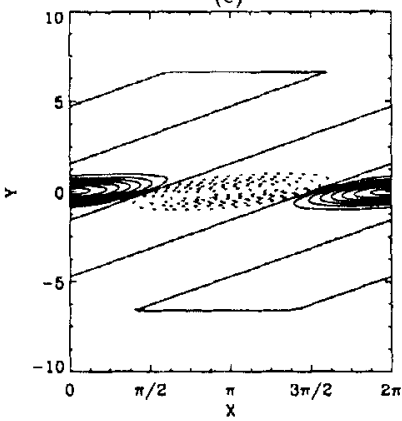

(h)

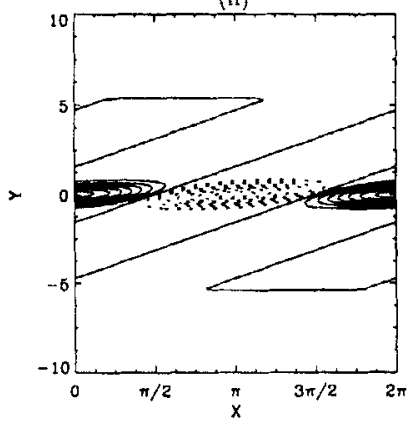

(c)
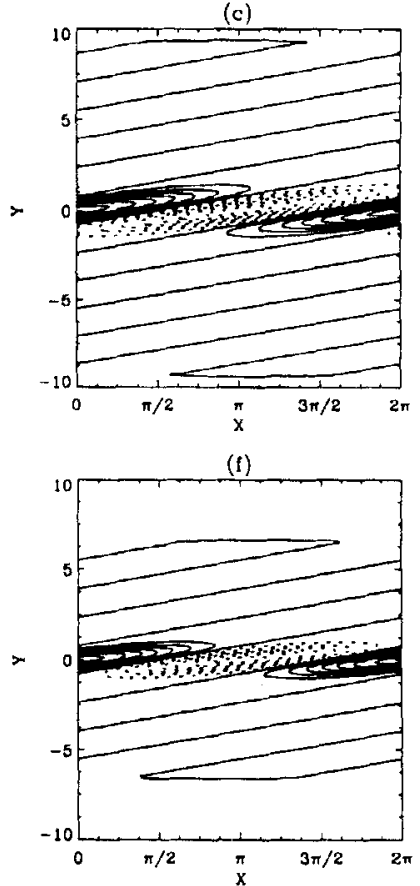

(i)

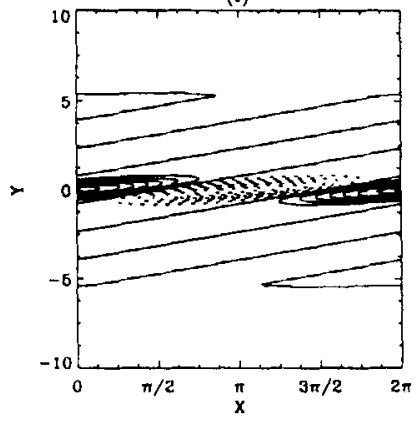

Figure 6. Perturbation vorticity for zonal wave-number $k=1$. Panels are the same as in Fig. 5. Contour interval is $1.33 \times 10^{-1} \mathrm{~s}^{-1}$ in all panels.

To complete the derivation, the inverse Fourier transform is applied to (3.2) giving

$$
\psi_{k}=\int_{a}^{b} G\left(y, y_{0}\right) \hat{\zeta}_{0} e^{\mathrm{i} k\left(x-t y_{0}\right)} \mathrm{d} y_{0},
$$

where the subscript $k$ is in accord with the convention of section 2 .

\section{(b) Uniform initial condition}

The initial vorticity given by $\hat{\zeta}_{0}=-k^{2}$, which corresponds to the upright plane wave of section 2 , is considered first. Substituting $\hat{\zeta}_{0}$ into (3.4) yields

$$
\begin{aligned}
\psi_{k} & =\frac{1}{1+t^{2}}[\cos \{k(x-t y)\} \\
& \left.+\frac{\sinh \{k(y-b)\} \cos \{k(x-t a)\}-\sinh \{k(y-a)\} \cos \{k(x-t b)\}}{\sinh \{k(b-a)\}}\right] .
\end{aligned}
$$




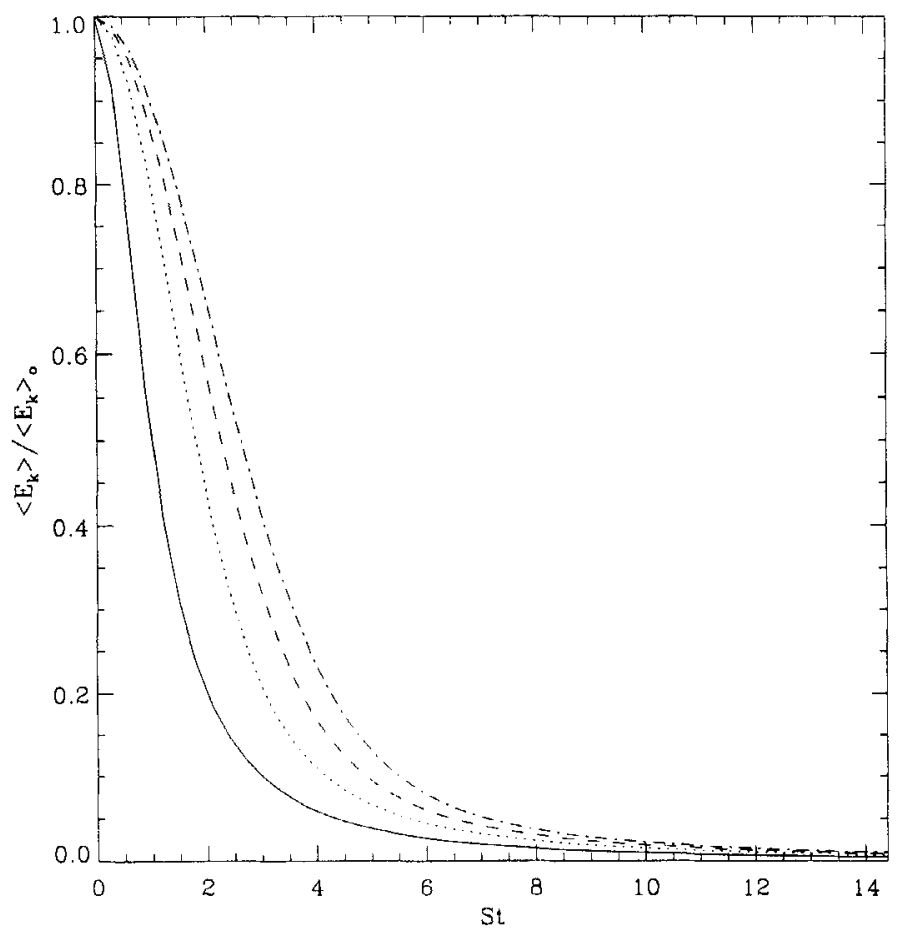

Figure 7. Normalized perturbation kinetic energy for zonal wave-number $k=1$ for the Gaussian initial vorticityprofiles $\hat{\zeta}_{0}=\exp \left(-y^{2}\right)$ (dot), $\hat{\zeta}_{0}=\exp \left(-2 y^{2}\right)$ (dash), and $\hat{\zeta}_{0}=\exp \left(-3 y^{2}\right)$ (dot dash). The solid curve is the normalized kinetic energy for the upright plane wave. Infinite channel.

The first term in (3.5) is recognized as the upright-plane-wave solution while the second term is the boundary correction that ensures $\psi_{k}(a)=\psi_{k}(b)=0$. It is seen from (3.5) that as $k$ increases and/or $b-a$ increases, the magnitude of the boundary correction decreases.

Disturbance evolution in a bounded channel may be further analysed by examining the integrated kinetic energy (KE) associated with (3.5). This is given by

$$
\begin{aligned}
\left\langle E_{k}\right\rangle & =\frac{k^{2}}{2\left(1+t^{2}\right)^{2}}\left(\frac{\left(1+t^{2}\right)}{2}+\frac{2[\cos \{k t(b-a)\}-\cosh \{k(b-a)\}]}{k(b-a) \sinh \{k(b-a)\}}\right. \\
& -\frac{\cos \{k t(b-a)\} \cosh \{k(b+a)\}\{\sinh (2 k b)-\sinh (2 k a)\}}{2 k(b-a) \sinh ^{2}\{k(b-a)\}} \\
& +\frac{\cos \{k t(b-a)\} \sinh \{k(b+a)\}\{\cosh (2 k b)-\cosh (2 k a)\}}{2 k(b-a) \sinh ^{2}\{k(b-a)\}} \\
& \left.+\frac{\sinh \{2 k(b-a)\}}{2 k(b-a) \sinh ^{2}\{k(b-a)\}}\right) .
\end{aligned}
$$

The first term in (3.6) is the kinetic energy of the upright plane wave while the other terms describe the boundary effects on the perturbation energy. Since the first term decays as $t^{-2}$ while the remaining terms decay as $t^{-4}$, the upright-plane-wave solution emerges for increasing $t$. Figure 8 shows the normalized KE as a function of time for initial vorticity $\hat{\zeta}_{0}=-k^{2}$ for boundaries at $a=-1, b=1$. Unlike the upright-plane-wave case, where the energy decay was independent of $k$, perturbations decay more rapidly with increasing wave-number when forced by a constant initial condition in a bounded channel. Moreover, the $k=1$ perturbation decays significantly more slowly than the higher-wave-number 
perturbations. While very different from the upright plane wave, disturbance evolution forced by a uniform profile of initial vorticity in bounded shear is qualitatively similar to that forced by a Gaussian profile of initial vorticity in unbounded shear.

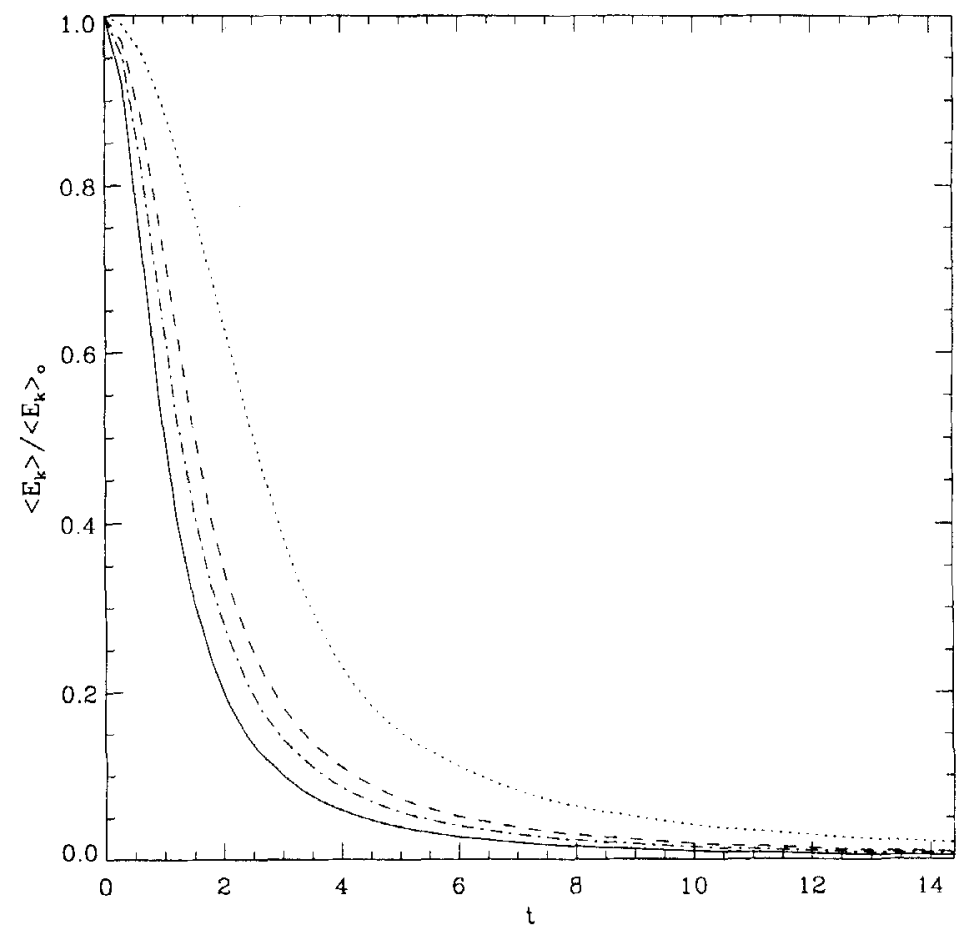

Figure 8. Normalized kinetic energy for uniform initial vorticity $\hat{\zeta}_{0}=-k^{2}$ and boundaries at $a=-1.0, b=1.0$. The curves show the energy decay for $k=1$ (dot), $k=2$ (dash) and $k=3$ (dot dash). The solid curve shows the energy decay for the upright plane wave in unbounded simple shear. Bounded channel.

\section{(c) Gaussian initial vorticity}

Perturbation decay forced by Gaussian profiles of initial vorticity in a bounded channel was also considered. Since (3.4) does not admit exact solutions, the trapezoidal rule was used to evaluate the integral. Inspection of the perturbation streamfunction- and vorticityfields for the initial condition $\hat{\zeta}_{0}=\exp \left(-y^{2}\right)$ and ZWN $k=1$ (not shown), showed that for $(b-a)<\alpha^{-1 / 2}$ the maximum streamfunction amplitude and decay-rate decreased relative to the unbounded case. For $(b-a)>\alpha^{-1 / 2}$, the unbounded decay-rate is recovered provided $b-a$ is sufficiently large to render boundary effects in the Green's function negligible.

\section{AXISYMMETRIZATION IN SWIRLING FLOW}

\section{(a) Introduction}

In this section we extend previous results to a rapidly rotating fluid with radiallyvarying shear. The flow model consists of a radially-bounded vortex, and the decay dependence on the AWN or radial structure of asymmetric profiles of initial vorticity is of particular interest. As in previous sections, the linear inviscid incompressible vorticityequation is employed, though here a cylindrical coordinate-system is chosen to represent the problem. 
When formulating a vortex model in cylindrical coordinates, one must decide if the system is ground-based or storm-relative. Based on the equations presented by Willoughby (1979), SM showed that in storm-relative coordinates, vortex motion on an $f$-plane merely modifies the definition of pressure while leaving the form of the momentum and thermodynamic equations intact. Since the model adopted below is formulated at the level of the vorticity equation, storm motion only appears explicitly in the boundary condition at infinity. In a Fourier azimuthal representation, storm motion projects onto the AWN-1 wind component. For the simple case of a resting environment, winds associated with wave number one must coincide with the motion at infinity while all other azimuthal components must vanish there. The more widely-used choice of coordinates is the ground-based system. Here, all wind asymmetries must vanish at infinity in a resting environment. Since we are primarily concerned with the physics of asymmetric decay and not vortex motion per se, a ground-based coordinate system will be adopted.

\section{(b) Swirling model}

The linearized vorticity-equation in cylindrical coordinates is given by

$$
\frac{\partial \zeta}{\partial t}+\frac{\bar{v}}{r} \frac{\partial \zeta}{\partial \lambda}+u \frac{\mathrm{d} \bar{\eta}}{\mathrm{d} r}=0
$$

where

$$
\zeta=\frac{1}{r} \frac{\partial(r v)}{\partial r}-\frac{1}{r} \frac{\partial u}{\partial \lambda}
$$

is the vertical component of perturbation relative vorticity, $(r, \lambda)$ are the radial and azimuthal coordinates, $\bar{v}$ denotes the basic-state tangential wind, $(u, v)$ are the radial and azimuthal perturbation winds, and $\bar{\eta}$ is the basic-state absolute vorticity. Equation (4.1) is non-dimensionalized by letting $r=R_{\mathrm{m}} \tilde{r}, \lambda=\tilde{\lambda},(u, v)=V_{\mathrm{m}}(\tilde{u}, \tilde{v}), t=R_{\mathrm{m}} \tilde{t} / V_{\mathrm{m}}$ and $\zeta=V_{\mathrm{m}} \tilde{\zeta} / R_{\mathrm{m}}$ where $V_{\mathrm{m}}$ is the maximum tangential wind at radius $R_{\mathrm{m}}$. Upon dropping tildes, the resulting non-dimensional vorticity-equation is identical to (4.1).

In simple-shear flow, perturbation decay was controlled by the meridional gradient of the basic-state zonal wind. It is therefore expected that the radial gradient of the basicstate angular velocity $(\bar{\Omega}=\bar{v} / r)$ will control disturbance decay in a swirling vortex. To first order, the angular-velocity gradient in a hurricane is approximately zero inside the radius of maximum winds. Since little symmetrization should occur there, a boundary is placed at the radius of maximum winds $r=a$. In keeping with the linear assumption, an environmental boundary is placed at $r=b$ such that $\bar{v} \gg(u, v)$. These boundaries are assumed to be slippery walls at which the normal velocity vanishes. Thus, $u(a)=u(b)=0$.

To satisfy continuity, a perturbation streamfunction is defined such that

$$
u=-\frac{1}{r} \frac{\partial \psi}{\partial \lambda} \quad v=\frac{\partial \psi}{\partial r}
$$

The vorticity equation (4.1) is then

$$
\left(\frac{\partial}{\partial t}+\frac{\bar{v}}{r} \frac{\partial}{\partial \lambda}\right)\left\{\frac{1}{r} \frac{\partial}{\partial r}\left(r \frac{\partial}{\partial r}\right)+\frac{1}{r^{2}} \frac{\partial^{2}}{\partial \lambda^{2}}\right\} \psi-\frac{1}{r} \frac{\mathrm{d} \bar{\eta}}{\mathrm{d} r} \frac{\partial \psi}{\partial \lambda}=0 .
$$

Assuming solutions of the form $\psi=\hat{\psi}(r, t) \exp (\mathrm{in} \lambda)$ and taking the Laplace transform of the resulting equation yields

$$
\left(q+\mathrm{in} \frac{\bar{v}}{r}\right)\left\{\frac{1}{r} \frac{\partial}{\partial r}\left(r \frac{\partial}{\partial r}\right)-\frac{n^{2}}{r^{2}}\right\} \hat{\psi}^{(q)}-\frac{\mathrm{in}}{r} \frac{\mathrm{d} \bar{\eta}}{\mathrm{d} r} \hat{\psi}^{(q)}=\left\{\frac{1}{r} \frac{\partial}{\partial r}\left(r \frac{\partial}{\partial r}\right)-\frac{n^{2}}{r^{2}}\right\} \hat{\psi}_{0}
$$


where $\hat{\psi}_{0}$ is the radial structure of the initial Fourier streamfunction amplitude for AWN $n$, and $q$ is the Laplace-transform parameter. Noting that the right side of (4.5) is the initial vorticity for wave number $n$ and upon dividing by $q+\mathrm{i} n \bar{v} / r$, equation (4.5) becomes

$$
\left[\frac{1}{r} \frac{\partial}{\partial r}\left(r \frac{\partial}{\partial r}\right)-\frac{n^{2}}{r^{2}}\right] \hat{\psi}^{(q)}-\frac{\mathrm{i} n}{r} \frac{\mathrm{d} \bar{\eta}}{\mathrm{d} r} \frac{\hat{\psi}^{(q)}}{(q+\mathrm{i} n \bar{v} / r)}=\frac{\hat{\zeta}_{o}}{(q+\mathrm{i} n \bar{v} / r)} .
$$

Unlike the rectilinear problem of sections 2 and 3 which possessed zero basic-state vorticity-gradient, the general solution to (4.6) is more complicated. As discussed in section 6 of Case (1960) for the analogous rectilinear problem, the inversion of (4.6) requires evaluation of contour integrals in the complex plane. On appealing to the calculus of residues the general solution consists of a sum of discrete exponentials (normal modes) associated with the zeros of the Wronskian in combination with an integral over branch cuts (continuous spectrum) associated with the zeros of $q+i n \bar{v} / r$. With the exception of a few known cases (e.g. section 5 of the present paper), closed-form solutions describing the explicit dynamics of both continuous-spectrum and normal-mode components are generally not available. Here, attention is restricted to a specific class of vortices for which simple solutions are possible. If the basic-state vorticity-gradient is identically zero (e.g. $\mathrm{CW}$ ), the solution to (4.6) is readily constructed. For the geometry considered here, the bounded Rankine profile given by

$$
\bar{v}=a / r, \quad a \leqslant r \leqslant b
$$

with $a \neq 0$ and $b$ finite satisfies this requirement since $\bar{\eta}(r)=f$. The inverse Laplace transform of (4.6) for the bounded Rankine vortex is then

$$
\left[\frac{\partial}{\partial r}\left(r \frac{\partial}{\partial r}\right)-\frac{n^{2}}{r}\right] \hat{\psi}=r \hat{\zeta}_{0} e^{-i a n t / r^{2}}
$$

The solution to (4.8) is

$$
\hat{\psi}(r)=\int_{a}^{b} G(r, \rho) \hat{\zeta}_{0}(\rho) e^{-\mathrm{i} a n t / \rho^{2}} \rho \mathrm{d} \rho,
$$

where the Green's function is given by

$$
G(r, \rho)=\frac{1}{2 n r^{n}\left(a^{2 n}-b^{2 n}\right)} \begin{cases}\left(\rho^{n}-b^{2 n} \rho^{-n}\right)\left(a^{2 n}-r^{2 n}\right), & a \leqslant r \leqslant \rho \\ \left(\rho^{n}-a^{2 n} \rho^{-n}\right)\left(b^{2 n}-r^{2 n}\right), & \rho \leqslant r \leqslant b .\end{cases}
$$

Following the convention of section 2, the Fourier inversion for wave number $n$ is

$$
\psi_{n}=e^{\mathrm{i} n \lambda} \int_{a}^{b} G(r, \rho) \hat{\zeta}_{0} e^{-\mathrm{i} a n t / \rho^{2}} \rho \mathrm{d} \rho
$$

Since exact solutions to (4.11) cannot generally be found, the trapezoidal rule is used throughout. Unless otherwise noted, the locations of the boundaries are set at $a=1$ and $b=10$. 


\section{(c) Initial conditions}

To gain insight into the asymmetric dynamics that are applicable to hurricanes and other geophysical vortices, initial vorticity-profiles are needed that represent physical processes relevant in such flows. CW proposed four classes of initial conditions that represent the effects of specific forced asymmetries occurring in hurricanes. These initial data are

$$
\begin{array}{ll}
\hat{\zeta}_{0}=-r^{-1} & n=1 \\
\hat{\zeta}_{0}=r^{-3} & n=1 \\
\hat{\zeta}_{0}=-i r^{-2} & n=2 \\
\hat{\zeta}_{0}=r^{-(n+4)} &
\end{array}
$$

$\mathrm{CW}$ interpreted these initial conditions as follows. Equation (4.12a) represents the generation of a wave-number-one asymmetry due to the advection of absolute environmental vorticity by the symmetric vortex, the $\beta$-effect. This includes the planetary $\beta$ as well as the meridional gradient of the environmental vorticity. Equation (4.12b) represents the generation of a wave-number-one asymmetry due to the vortex motion relative to the environmental wind, while (4.12c) represents the generation of a wave-number-two asymmetry due to the linear shearing of the symmetric vorticity by the relative environmental wind. CW presented the initial vorticity in (4.12d) to obtain analytical solutions to the integral in (4.11). These profiles may be associated with convection in hurricane-eye walls. CW named these profiles of initial vorticity the 'beta-induced', 'motion-induced', 'shear-induced' and 'convection-induced' asymmetries respectively.

\section{(d) Model results}

Since one of the objectives of this work was to clarify the CW paper further, attempts were made to reproduce their results. Although their streamfunction fields were easily reconstructed, integrated kinetic-energy plots associated with the four initial conditions developed in the previous subsection were found to be subtly different from those presented by $\mathrm{CW}$. Specifically, CW showed a more rapid energy-decay for convection-induced initial conditions and a more abrupt transition to the asymptotic long-time behaviour. The conclusions $\mathrm{CW}$ drew from their figure, however, are essentially qualitative in nature and are readily obtained from our results (not shown).

In their paper, $\mathrm{CW}$ restricted attention to the profiles of initial vorticity developed in the previous section. While physically motivated, both the radial structure and the AWN are changed simultaneously. To isolate the disturbance decay-dependence on either of these factors, initial vorticity-profiles based on those of $\mathrm{CW}$ are again considered but only one parameter is changed at a time.

Figure 9 shows the streamfunction fields for an upright distribution of initial vorticity $\hat{\zeta}_{0}=r^{-3}$ and Fig. 10 shows the corresponding vorticity fields. The rows represent wavenumbers $n=1,2$ and 3 respectively, while the columns designate times $t=0.0,3.6$ and 7.2 respectively. These figures show that, with respect to increasing the time or the wave number, the rectilinear and swirling models give similar results. This is not surprising as the Green's function and the phase function are formally identical in the rectilinear and swirling models.

While the mathematical solutions are qualitatively similar in the rectilinear and swirling models, there are physical differences in the disturbance evolution between the two systems. Figure 11 shows the energy decay in the rectilinear model for the initial condition $\hat{\zeta}_{0}=(11-y)^{-3}$. Figure 12 shows the energy decay in the swirling model for $\hat{\zeta}_{0}=r^{-3}$. These plots show that, on a wave-number by wave-number basis, the energy decays more 
(a)

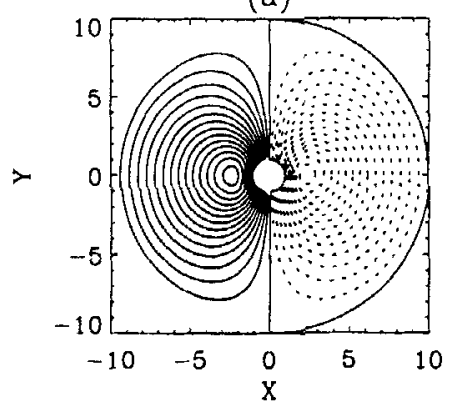

(d)

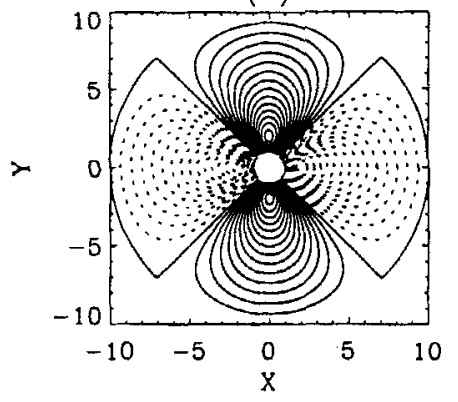

(g)

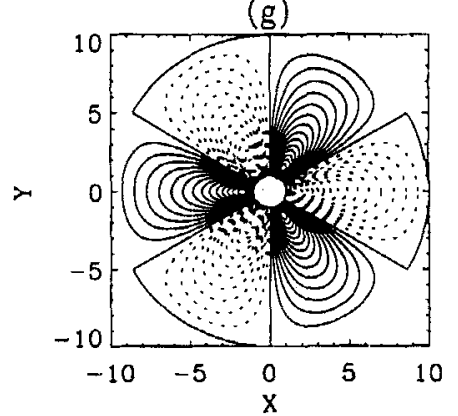

(b)

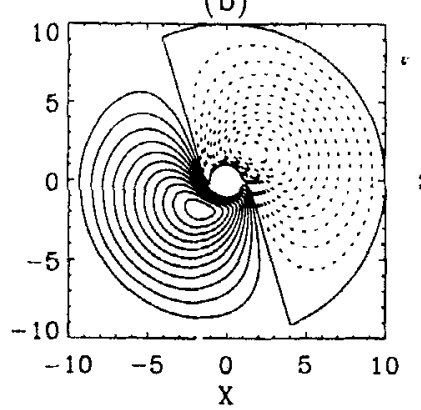

(e)

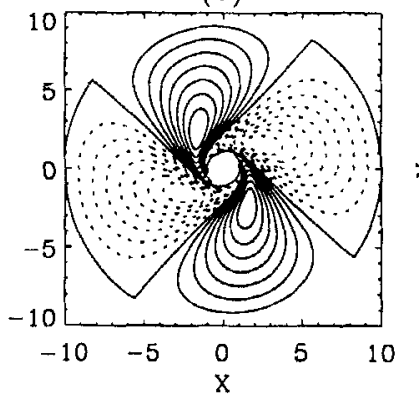

(h)

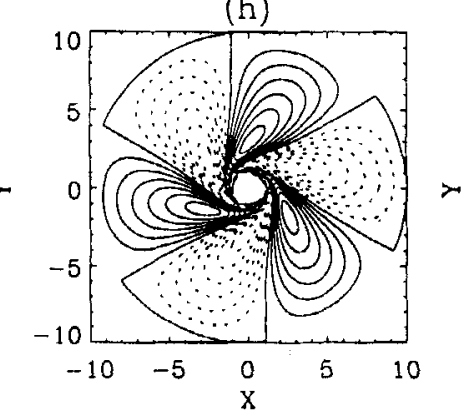

(c)

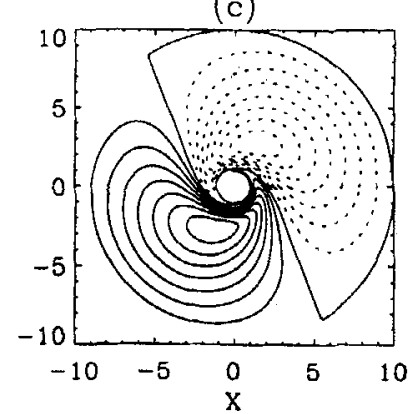

(f)

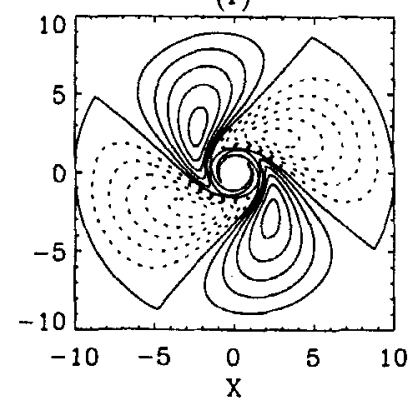

(i)

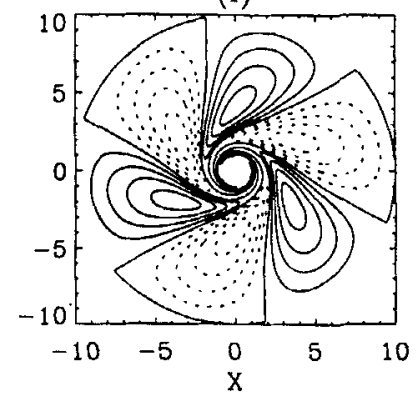

Figure 9. Perturbation streamfunction field for $\hat{\zeta}_{0}=r^{-3}$ for the bounded Rankine vortex; $a=1, b=10$. The columns show contours at times $t=0.0, t=3.6$ and $t=7.2$ respectively. Panels (a)-(c) show contours for $n=1$ where the contour interval is $1.10 \times 10^{-2}$. Panels (d) $-(\mathrm{f})$ show contours for $n=2$ where the contour interval is $5.67 \times 10^{-3}$. Panels $(\mathrm{g})$-(i) show contours for $n=3$ where the contour interval is $3.31 \times 10^{-3}$.

slowly in the swirling model than in the rectilinear one. In addition, the figures suggest that the difference between the wave-number-one disturbance and the higher-wave-number disturbances is more pronounced in the swirling case. These differences are explained by examining how the shear acts in each system.

For the rectilinear model considered in section 2 , the shear was constant and defined limiting values for the decay of perturbation kinetic-energy. Specifically, the limiting energy-decay was given by $\left\{1+(S t)^{2}\right\}^{-1}$ and the limiting time for the half life of a perturbation was $t=S^{-1}$. In the swirling case, however, the shear varies radially and is only a local measure of the flow. In order to investigate the limiting integrated energy-decay of asymmetries, a global measure of the shear is desired.

In simple shear and in the bounded Rankine vortex, the physical process governing disturbance evolution is the conservation of vorticity following the basic-state flow. In 
(a)

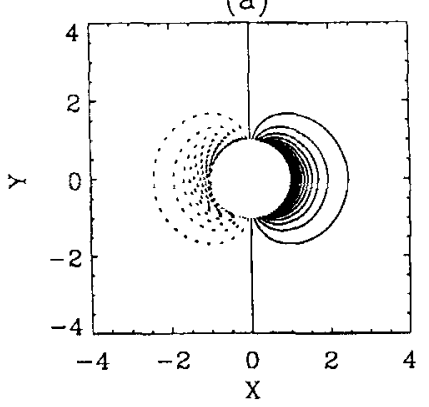

(d)

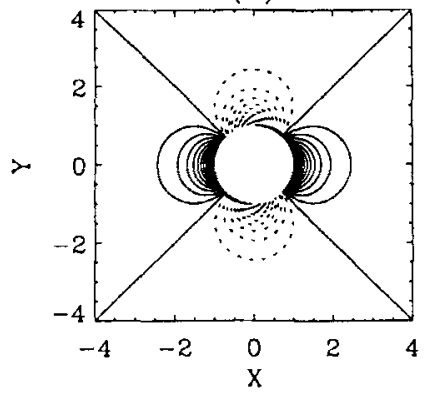

(g)

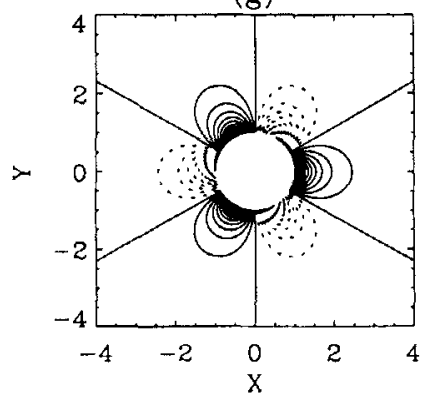

(b)

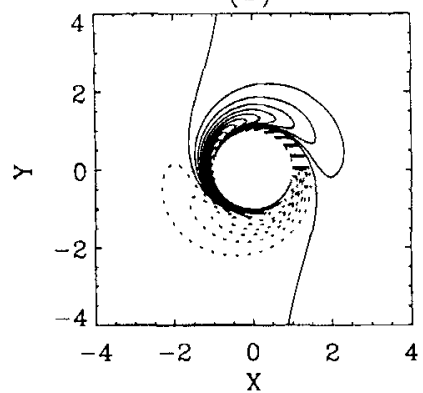

(e)

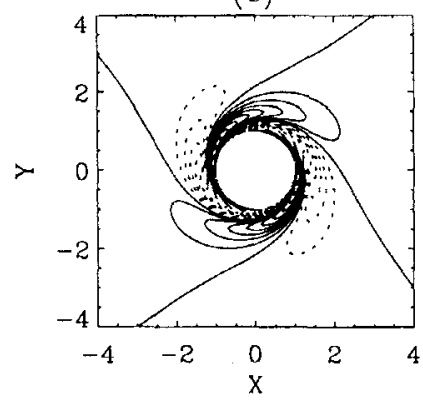

(h)

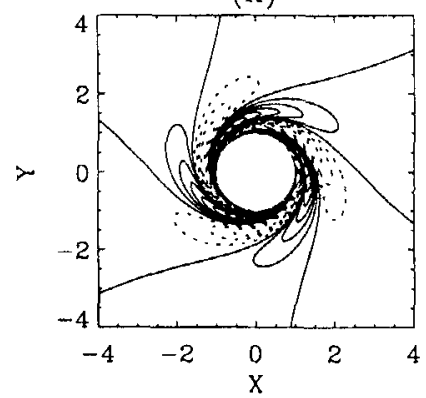

(c)

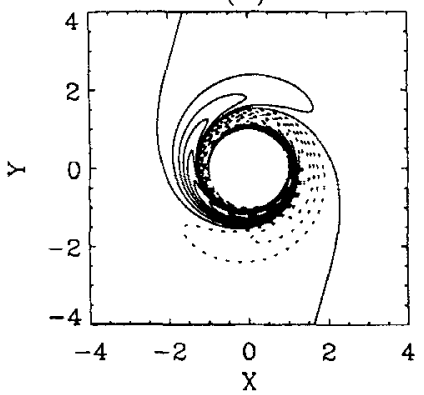

(f)

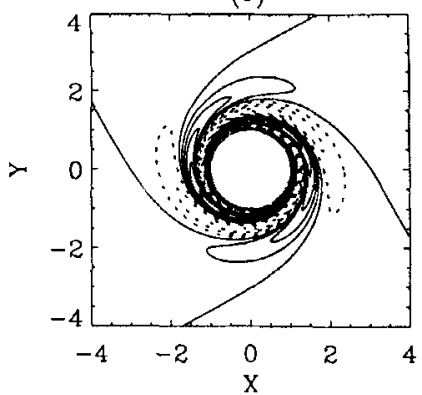

(i)

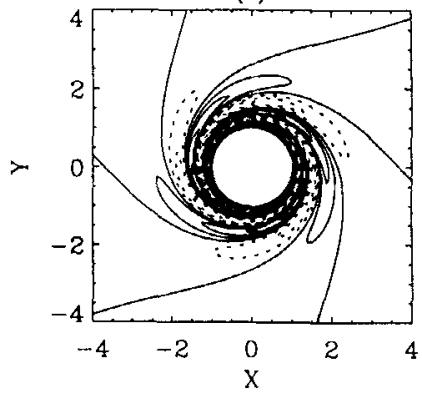

Figure 10. Perturbation vorticity-field corresponding to Fig. 9. The contour interval is $6.90 \times 10^{-2}$.

the rectilinear model of section 2 , the perturbation vorticity is advected by $\bar{u}$, the basicstate zonal wind. The corresponding shear is thus $\mathrm{d} \bar{u} / \mathrm{d} y$. Since the shear is constant, the meridional scale of the perturbation qualitatively describes the decay rate for a constant ZWN. The change in the zonal wind across a large-scale perturbation is greater than the corresponding change across a small-scale perturbation. Thus, the large-scale disturbances decay more rapidly.

In the swirling case, the disturbance vorticity is advected by $\bar{v} / r$, the basic-state angular velocity. The corresponding local shear is $r \mathrm{~d} \bar{\Omega} / \mathrm{d} r$ (SM) where $\bar{\Omega}=\bar{v} / r$. In a Rankine vortex, $\bar{\Omega}$ is equal to $r^{-2}$ and the local shear is equal to $-2 r^{-2}$. Since the angular velocity does not describe solid-body rotation, both the radial location and the radial structure of the asymmetry are needed to describe the decay rate. An effective shear that satisfies these conditions is

$$
S_{\mathrm{eff}}=\frac{\int_{a}^{b} r \frac{\mathrm{d} \bar{\Omega}}{\mathrm{d} r} \hat{\zeta}_{0} d r}{\int_{a}^{b} \hat{\zeta}_{0} \mathrm{~d} r}
$$




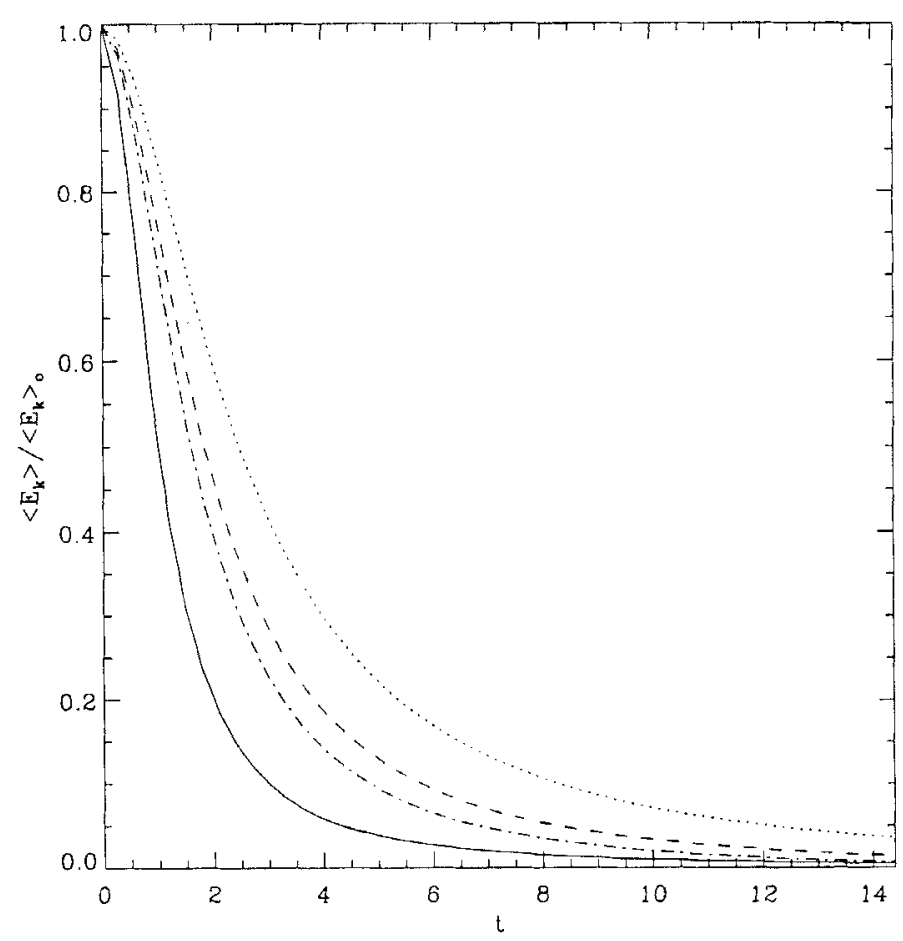

Figure 11. Normalized kinetic energy for the bounded rectilinear model with $\hat{\zeta}_{0}=(11-y)^{-3} ; a=1, b=10$. The curves show the energy decay for: $k=1$ (dot), $k=2$ (dash), $k=3$ (dot dash). The solid curve shows the energy decay for the upright plane wave given by $1 /\left(1+t^{2}\right)$ in unbounded simple shear.

The analogous expression in the rectilinear model reduces to the simple shear definition if $\mathrm{d} \overline{\boldsymbol{u}} / \mathrm{d} y$ is constant. For initial conditions of the form $r^{-\alpha}$, the effective shear is found to be

$$
\begin{gathered}
S_{\text {eff }}=-\frac{2(\alpha-1)}{a^{2} b^{2}(\alpha+1)}\left(\frac{a^{\alpha+1}-b^{\alpha+1}}{a^{\alpha-1}-b^{\alpha-1}}\right), \quad \alpha \neq 1 \\
S_{\text {eff }}=\frac{a^{2}-b^{2}}{a^{2} b^{2} \ln (b / a)}, \quad \alpha=1 .
\end{gathered}
$$

Based upon the rectilinear model, the effective shear should define limiting values for the decay rate of asymmetries. In particular, the limiting energy-decay should be given by $\left\{1+\left(S_{\text {eff }} t\right)^{2}\right\}^{-1}$. Thus, the limiting asymmetry half-life should be $t=-S_{\text {eff }}^{-1}$. The limiting energy-decay curve is the solid line in Fig. 12.

Figure 13 shows the integrated energy as a function of time for AWN $n=1$ and vorticity profiles $\hat{\zeta}_{0}=r^{-\alpha}$ where $\alpha=3,4,5$ and 6 . Figure 13 confirms the expected behaviour for the $r^{-3}$ and $r^{-4}$ asymmetries. However, the decay rates for the $r^{-5}$ and $r^{-4}$ asymmetries are nearly identical until $t \approx 5.5$. In addition, the decay rate for the $r^{-6}$ asymmetry is less than that for the $r^{-4}$ asymmetry until $t \approx 8.0$ and is less than the decay rate for the $r^{-5}$ asymmetry for the times plotted. Thus, for initial conditions tightly confined to the inner boundary, there is a transient period when the decay rate decreases with decreasing disturbance-scale. This result resembles that of the rectilinear model. The decay rate eventually reverts back to increasing with decreasing disturbance-scale though the cross-over time is later for smaller-scale disturbances. 


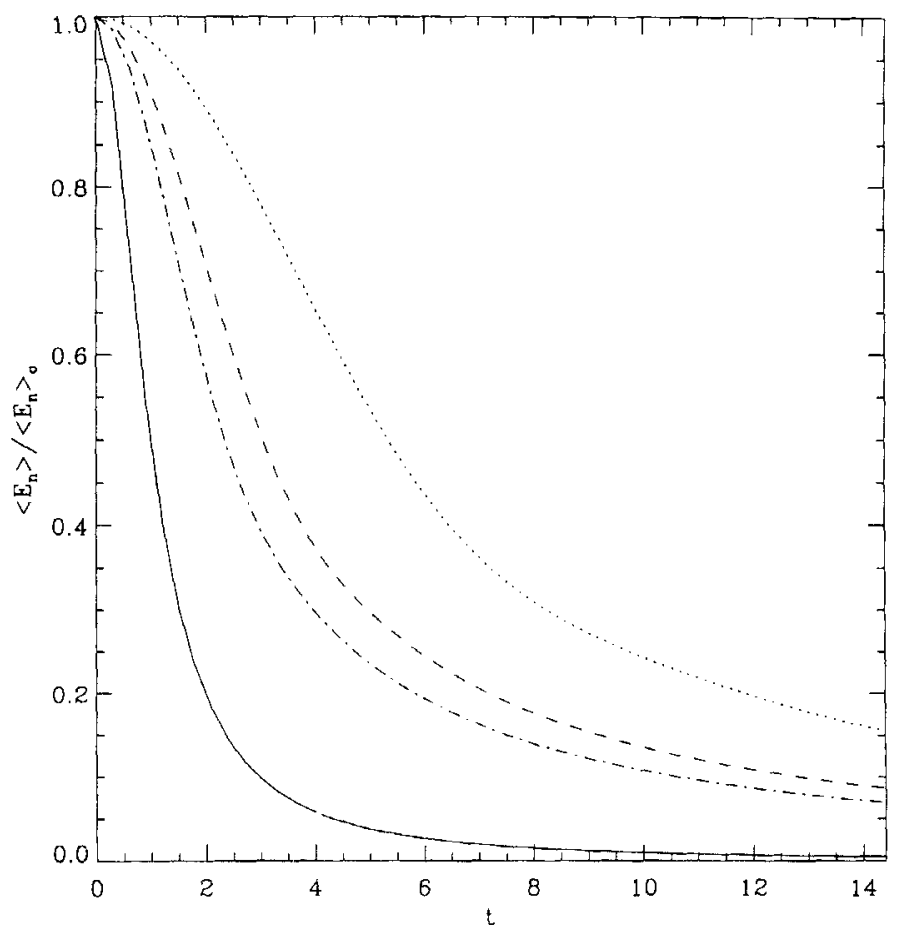

Figure 12. Normalized kinetic energy for the bounded Rankine vortex with $\hat{\zeta}_{0}=r^{-3} ; a=1, b=10$. The curves show the energy decay for: $n=1$ (dot), $n=2$ (dash), $n=3$ (dot dash). The solid curve shows the limiting energy decay given by $1 /\left[1+\left(S_{\text {eff }} t\right)^{2}\right]$ where $S_{\text {eff }}=-1.01$.

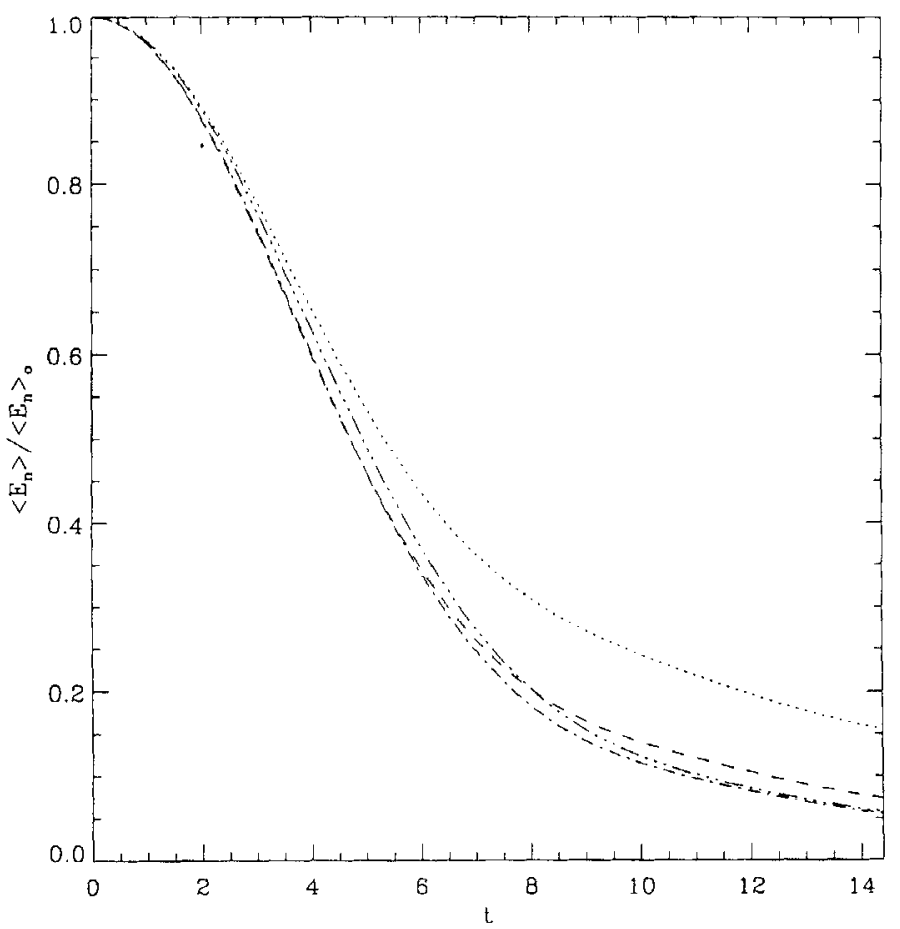

Figure 13. Normalized kinetic energy for the bounded Rankine vortex for $n=1 ; a=1, b=10$. The curves are the energy decay for: $\hat{\zeta}_{0}=r^{-3}$ (dot), $\hat{\zeta}_{0}=r^{-4}$ (dash), $\hat{\zeta}_{0}=r^{-5}$ (dot dash), and $\hat{\zeta}_{0}=r^{-6}$ (dot dot dot dash). 
This duality may be qualitatively explained as follows. Initially, most of the KE in the asymmetry is concentrated near the inner boundary. The radial change in the energy is so large that the basic-state shear appears constant in a first approximation. Thus, as in the rectilinear model, decreasing the disturbance scale results in a decrease of the decay rate. However, the inner portion of the asymmetry is symmetrized more rapidly than that farther out in the vortex. The KE near the inner boundary is transferred to the basic state much faster than that in the storm's environment. The cross-over time occurs when the inner-core energy decays to the same magnitude as the environmental energy. At this time, the basic-state shear no longer appears constant. The cross-over time is later for smallerscale disturbances because it takes longer for the inner-core KE to decay to environmental $\mathrm{KE}$ values.

The initial vorticity-profiles considered thus far represent forcing processes that act nearly continuously in a hurricane. It is also of interest to investigate transient forcings that act in hurricanes, such as an upper-level short-wave trough or a tropical easterly wave. As a proxy for such forcings, consider a Gaussian initial vorticity-profile of fixed radial scale. As the vorticity maximum is moved toward the vortex centre, the effective shear and the decay rate increase because of the increase in the differential rotation.

The Gaussian initial vorticity-profiles considered in this paper have all been initially upright with respect to the local shear. Consequently, such disturbances have only had a decay phase. However, migratory short-wave troughs, upon encountering a tropical cyclone, are generally not upright with respect to the local shear. As an example, consider a trough positioned $300 \mathrm{~km}$ east of a tropical cyclone in the northern hemisphere. Let the trough axis be oriented 30 degrees east of north such that the trough leans against the local shear. Recall from section 2 that disturbances leaning against the shear grow at the expense of the basic state. Thus, in the absence of competing processes, while the trough leans against the shear the trough will tend to intensify and the tropical cyclone will tend to weaken. As the trough grows, it rotates clockwise and becomes maximum when it is oriented east-west. Once the trough is upright with respect to the local shear, further evolution results in a weakening trough and an intensifying tropical cyclone.

Figures 14 and 15 show the asymmetry half-lives for profiles of initial vorticity of $r^{-2}$ and $r^{-5}$. For each profile, the $n=1$ asymmetry clearly decays more slowly than all higher-wave-number asymmetries. The dotted line in each plot is the asymmetry half-life $-S_{\text {eff }}^{-1}$ for the given initial condition. For the lower wave-numbers, $-S_{\text {eff }}^{-1}$ significantly underestimates the asymmetry half-life. For the $r^{-5}$ profile, the $-S_{\text {eff }}^{-1}$ line adequately defines the asymptotic limit of the half-life for the higher wave-numbers. The asymmetry half-life plot for the $r^{-3}$ vorticity profile (not shown) is similar to that for the $r^{-5}$ profile. This suggests that the above definition for $S_{\text {eff }}$ should be applicable to asymmetries which fall off in radius more rapidly than the basic-state angular velocity. This conclusion has been verified for Gaussian initial conditions.

The above results refute a suggestion made by $\mathrm{CW}$. Based on closed-form solutions obtained for the convection-induced asymmetries (cf. CW Eqs. (30) and (31)), CW argue that the asymmetry damping-rate should be proportional to the square of the AWN. If their assertion is correct, then as $n \rightarrow \infty$, the damping rate should also become infinite. Thus, the half-life should approach zero. However, plots of the asymmetry half-life show that for increasing $n$, the time required for the energy to decay to half its initial value approaches a non-zero constant and does not decrease as $n^{-2}$.

\section{(e) Application to hurricanes}

The definition for the effective shear presented in the previous section need not be restricted to the Rankine vortex. Here the effective shear is evaluated for the analytical 


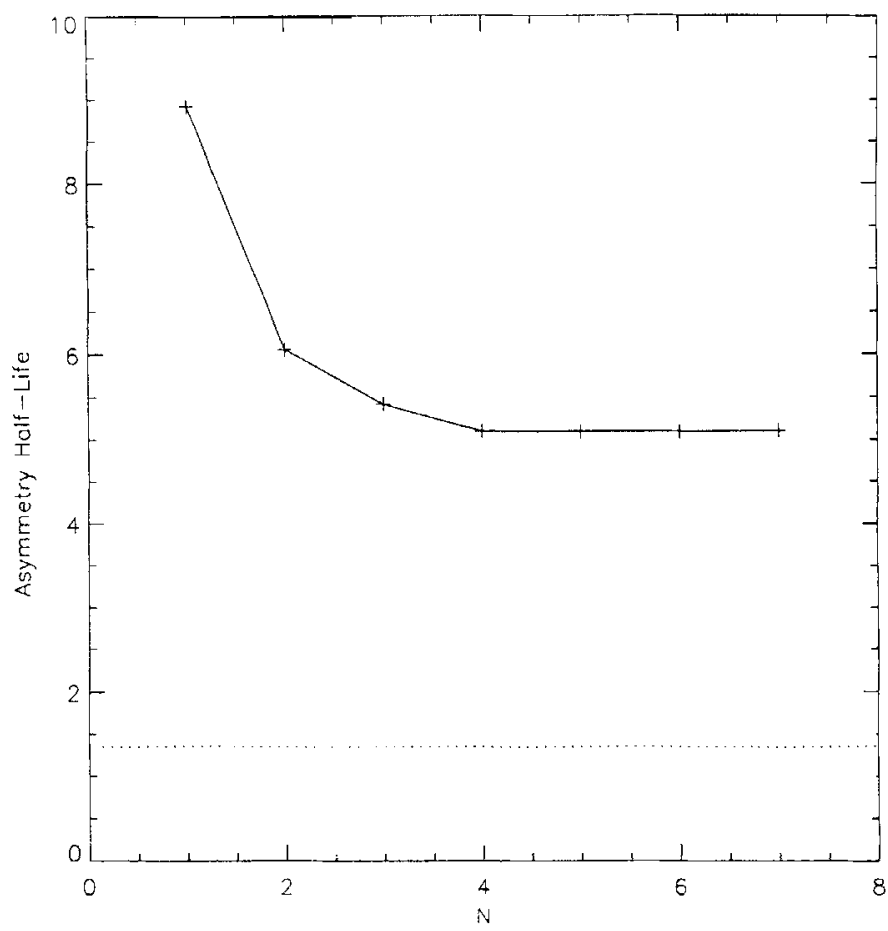

Figure 14. Time required for an asymmetry to decay to half its initial energy as a function of wave number $n$ for $\hat{\zeta}_{0}=r^{-2}$. The dotted line is the limiting case given by $-S_{\text {eff }}^{-1}$ where $S_{\text {eff }}=-0.74$. Bounded vortex: $a=1, b=10$.

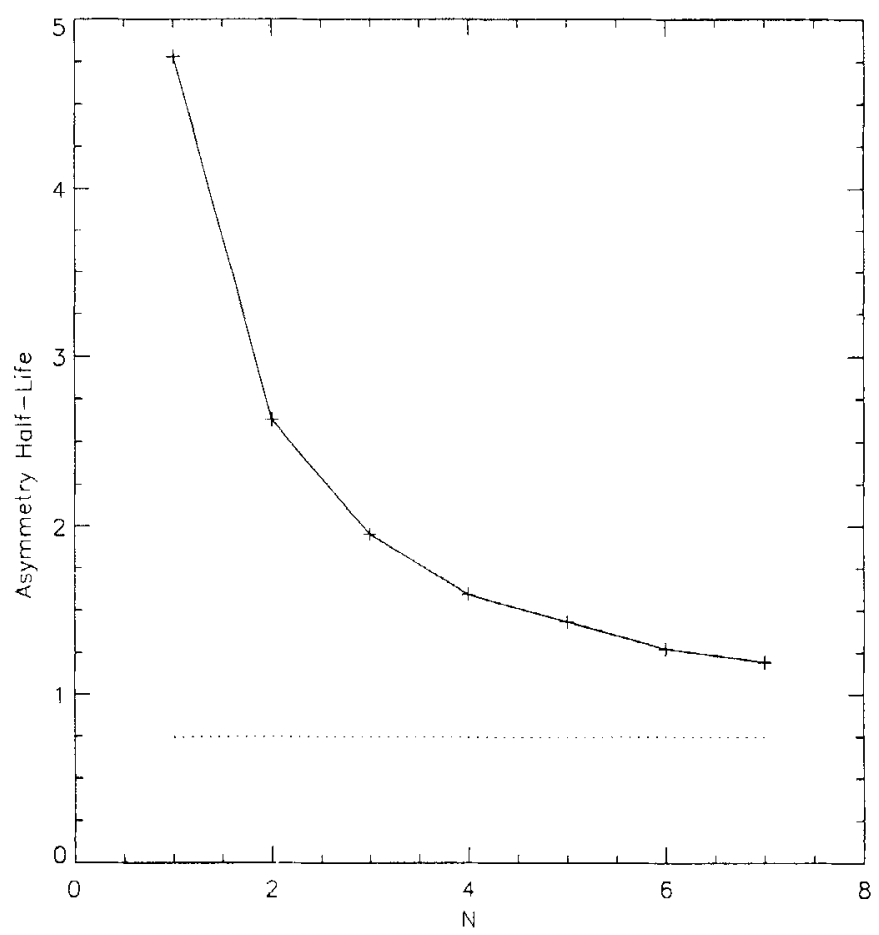

Figure 15. As Fig. 14 except $\hat{\zeta}_{0}=r^{-5}$ and $S_{\text {eff }}=-1.34$. 
profiles $\bar{v}=r^{-\alpha}$ where $\alpha=\frac{1}{3}, \frac{1}{2}$ and $\frac{2}{3}$. These profiles are better approximations of the symmetric tangential winds typically observed in hurricanes than is the Rankine vortex. The effective shear is also calculated for the observed $700 \mathrm{mb}$ winds in hurricane Gloria which occurred in 1985 . The Gloria data are non-dimensionalized using $V_{\mathrm{m}}=57.91 \mathrm{~m} \mathrm{~s}^{-1}$ and $R_{\mathrm{m}}=20 \mathrm{~km}$ corresponding to the hurricane's maximum tangential wind and radius of maximum winds, respectively. The Gloria winds are given at $10 \mathrm{~km}$ intervals and are considered out to $500 \mathrm{~km}$ from the storm centre. Thus, for a non-dimensional inner boundary set at $a=1$, the corresponding outer boundary occurs at $b=25$. Figure 16 shows the observed and analytical tangential winds.

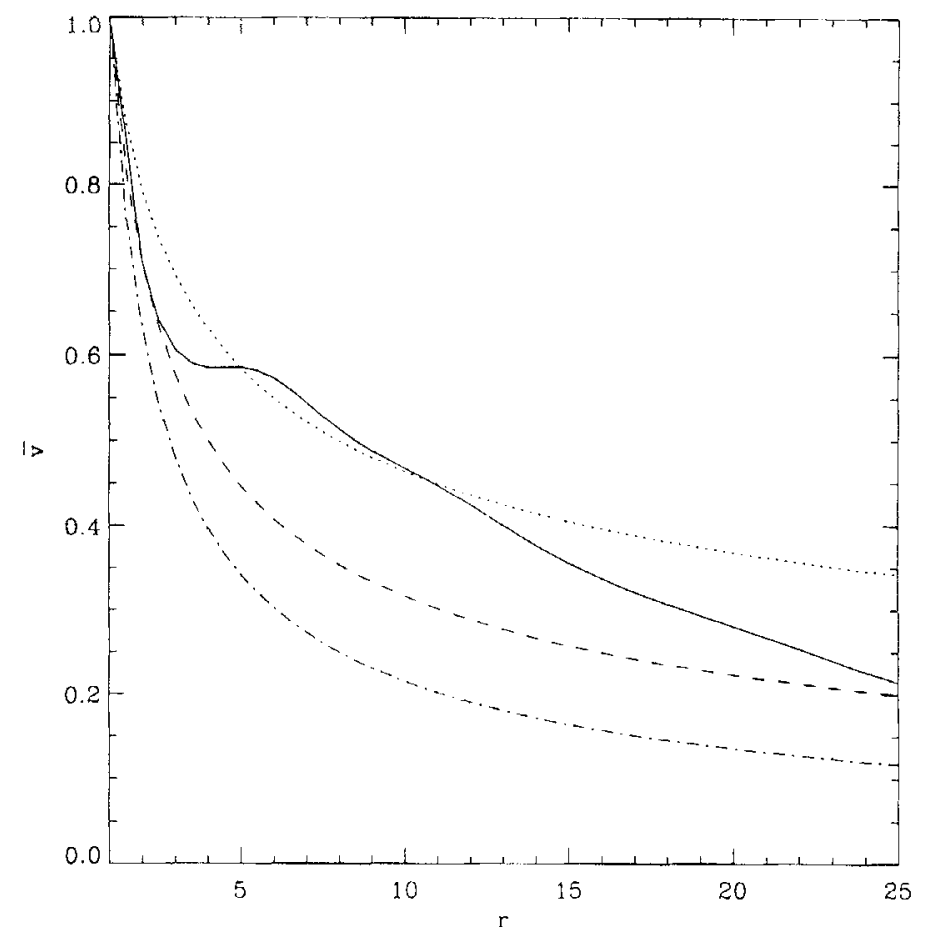

Figure 16. The non-dimensionalized Gloria and analytical tangential-wind profiles. The analytical winds are given by $\bar{v}=r^{-\alpha}$ where $\alpha=1 / 3$ (dot), 1/2 (dash) and $2 / 3$ (dot dash).

Since the effective-shear calculation also requires the specification of initial vorticityprofiles, three types of initial conditions are considered. The first is a convectively-forced asymmetry and is confined tightly to the radius of maximum winds. The second is shearinduced asymmetry which is also confined to the inner boundary but extends farther out into the vortex than the convection-induced asymmetry. The final initial condition corresponds to a trough in the environment of the vortex several hundred kilometres from the vortex centre. Mathematically, these initial conditions are given by $\hat{\zeta}_{0}=r^{-5}, r^{-2}$ and $\exp \left(-(r-15)^{2} / 10\right\}$ respectively.

Table 1 shows values of $S_{\text {eff }}$ and $-S_{\text {eff }}^{-1}$ for the profiles of initial vorticity and symmetric wind above. The values have been dimensionalized using the Hurricane Gloria data cited above. The asymmetry half-lives given by $-S_{\text {eff }}^{-1}$ are underestimates of the decay rate. Examination of Figs. 14 and 15, as well as the corresponding results for the $r^{-3}$ profile, indicate that the $n=1$ asymmetry decays approximately seven times more slowly than the limiting time-scale. Assuming that the $n=1$ decay rate approximates the decay rate for an atmospheric disturbance, asymmetries forced in the inner region of a hurricane would give 
up half their initial energy in 35-70 minutes for the analytical symmetric winds. The table suggests that similar asymmetries in Hurricane Gloria would have given up half their initial energy in 40-60 minutes. For the Gaussian initial vorticity considered here, representing a trough $300 \mathrm{~km}$ from the storm centre, disturbance half-lives would have been 17-35 hours for the analytical tangential-wind profiles. The table suggests that similar asymmetries in Hurricane Gloria would have given up half their initial energy in 14 hours.

TABLE 1. EFFECTIVE-SHEAR VALUES AND LIMITING ASYMMETRY HALF-LIVES

\begin{tabular}{|c|c|c|c|}
\hline \multirow{2}{*}{$\begin{array}{c}\text { Symmetric wind } \\
\text { profiles } \bar{v}\end{array}$} & \multicolumn{3}{|c|}{ Initial vorticity-profiles $\hat{\zeta}_{0}$} \\
\hline & $r^{-5}$ & $r^{-2}$ & $\exp \left\{-(r-15)^{2} / 10\right\}$ \\
\hline$r^{-1 / 3}$ & $\begin{array}{c}-2.81 \times 10^{-3} \mathrm{~s}^{-1} \\
5.94 \mathrm{~min}\end{array}$ & $\begin{array}{c}-1.77 \times 10^{-3} \mathrm{~s}^{-1} \\
9.44 \mathrm{~min}\end{array}$ & $\begin{array}{c}-1.09 \times 10^{-4} \mathrm{~s}^{-1} \\
2.56 \mathrm{hr}\end{array}$ \\
\hline$r^{-1 / 2}$ & $\begin{array}{c}-3.06 \times 10^{-3} \mathrm{~s}^{-1} \\
5.45 \mathrm{~min}\end{array}$ & $\begin{array}{c}-1.86 \times 10^{-3} \mathrm{~s}^{-1} \\
8.96 \mathrm{~min}\end{array}$ & $\begin{array}{c}-7.83 \times 10^{-5} \mathrm{~s}^{-1} \\
3.55 \mathrm{hr}\end{array}$ \\
\hline$r^{-2 / 3}$ & $\begin{array}{c}-3.29 \times 10^{-3} \mathrm{~s}^{-1} \\
5.07 \mathrm{~min}\end{array}$ & $\begin{array}{c}-1.94 \times 10^{-3} \mathrm{~s}^{-1} \\
8.58 \mathrm{~min}\end{array}$ & $\begin{array}{c}-5.59 \times 10^{-5} \mathrm{~s}^{-1} \\
4.97 \mathrm{hr}\end{array}$ \\
\hline Gloria & $\begin{array}{c}-2.89 \times 10^{-3} \mathrm{~s}^{-1} \\
5.76 \mathrm{~min}\end{array}$ & $\begin{array}{c}-1.81 \times 10^{-3} \mathrm{~s}^{-1} \\
9.23 \mathrm{~min}\end{array}$ & $\begin{array}{c}-1.29 \times 10^{-4} \mathrm{~s}^{-1} \\
2.15 \mathrm{hr}\end{array}$ \\
\hline
\end{tabular}

Note: Effective-shear values and limiting asymmetry half-lives for various symmetric wind profiles. Symmetric-wind data from the $700 \mathrm{mb}$ level of Hurricane Gloria are also considered. All values have been dimensionalized using Gloria's maximum azimuthal wind $\left(57.91 \mathrm{~m} \mathrm{~s}^{-1}\right)$ and the radius of maximum winds $(20 \mathrm{~km})$.

\section{AXISYMMETRIZATION IN UNBOUNDED RANKINE FLOW}

In this section, the asymmetric initial-value dynamics of an unbounded Rankine vortex is considered. Although the normal-mode dispersion relation for small-amplitude oscillations of a columnar vortex is well known (Thomson 1880; Lamb 1932, sect. 158), extension to include rotational disturbances throughout the vortex has not yet been implemented. In the context of swirling-flow dynamics, such a model is useful for illustrating the partitioning of normal-mode and continuous-spectrum solution-components. The vortex model constructed below is readily extended to multiple regions. In addition, the model admits an analogous rectilinear formulation (not shown) that should also prove useful when interpreting the linearized-filamentation dynamics of constant-vorticity layers (Pullin 1981; Dritschell 1988; Pullin et al. 1989; Saffman 1992).

The non-dimensional linearized vorticity-equation from section 4 still applies. The inner boundary is now at the origin while the outer boundary is removed to infinity. It is assumed that all quantities are bounded at the origin and vanish at infinity. When including the region inside the radius of maximum winds, the Rankine profile is given by

$$
\bar{v}= \begin{cases}r / a, & r \leqslant a \\ a / r, & r \geqslant a\end{cases}
$$

where $a$ is the non-dimensional radius of maximum winds and $r$ is the non-dimensional radius. The corresponding profile of basic-state vorticity is

$$
\bar{\zeta}= \begin{cases}2 / a, & r<a \\ 0, & r>a\end{cases}
$$

while the profile of absolute vorticity is given by

$$
\bar{\eta}= \begin{cases}R o^{-1}+2 / a, & r<a \\ R o^{-1}, & r>a .\end{cases}
$$


The quantity $R o^{-1}$ is the inverse Rossby number defined by $R o^{-1}=f R_{\mathrm{m}} / V_{\mathrm{m}}$ where $f$ is the Coriolis parameter and $V_{\mathrm{m}}$ is the symmetric wind at the radius of maximum winds $R_{\mathrm{m}}$. Figure 17 shows $\bar{v}$ and $\bar{\eta}$ for the full Rankine vortex.
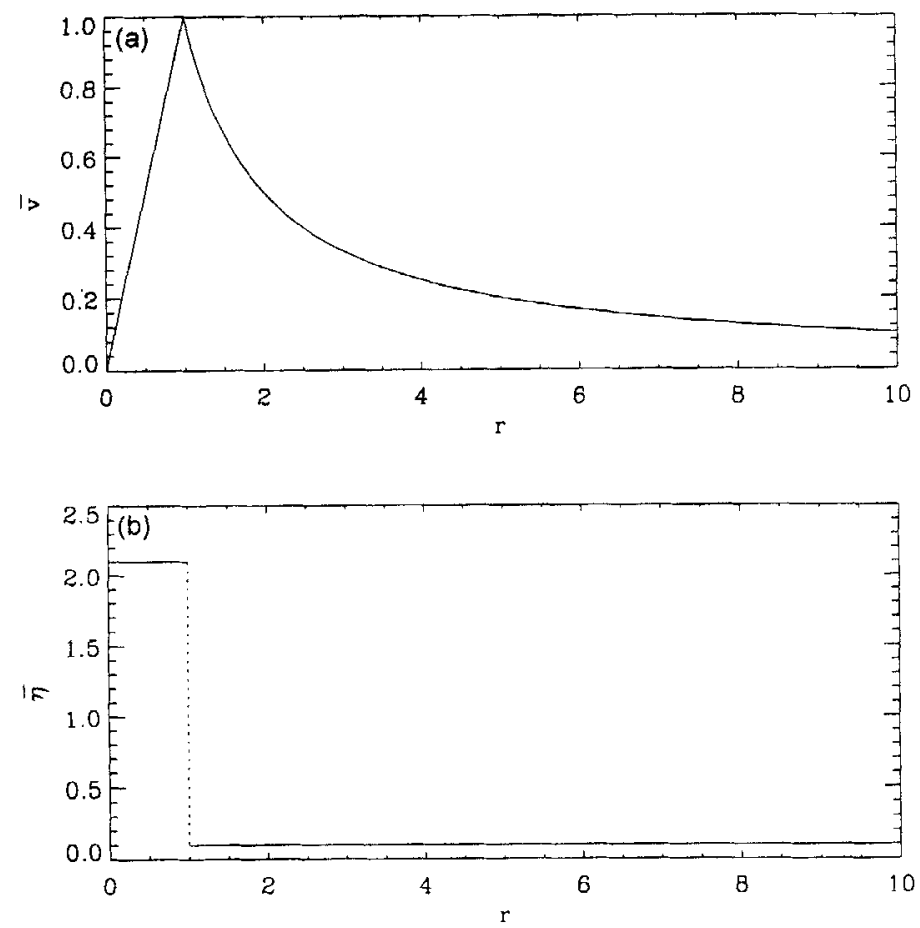

Figure 17. (a) Symmetric tangential winds $\bar{v}$ for the full Rankine vortex; (b) corresponding absolute vorticity using an inverse Rossby number of 0.1 .

The discontinuity in the mean-state vorticity at $r=a$ effectively introduces another boundary to the system. Since this boundary is interior to the fluid, the kinematic and dynamic boundary conditions must be satisfied at the disturbed interface $r=a+\epsilon$, where $\epsilon$ is the interface displacement. The kinematic boundary condition requires that the normal velocity be continuous while the dynamic boundary condition requires that the pressure be continuous at $r=a+\epsilon$. Consistent with the linearization employed below, the matching conditions will be evaluated at $r=a$. Once $u$ is determined, the evolution of the disturbed interface may be found upon integrating

$$
\left(\frac{\partial}{\partial t}+\frac{\bar{v}}{r} \frac{\partial}{\partial \lambda}\right) \epsilon=u
$$

for $\epsilon$ at $r=a$.

For the full Rankine profile (5.2) the linearized vorticity-equation of section 4 is modified to

$$
\left(\frac{\partial}{\partial t}+\frac{\bar{v}}{r} \frac{\partial}{\partial \lambda}\right) \zeta=0 \quad r \neq a .
$$

To solve (5.5), the discontinuity in the basic-state vorticity at $r=a$ must be accounted for. Since the problem is linear, the superposition principle may be used to separate the solution into two parts by letting $\zeta=\zeta_{\mathrm{s}}+\zeta_{1}$, where $\zeta_{\mathrm{s}}$ is defined to be smooth for all $r$ and $\zeta_{1}$ accounts for the discontinuity in the basic-state vorticity at $r=a$. The vorticity equation 
(5.5) is then split into two parts

$$
\begin{aligned}
& \left(\frac{\partial}{\partial t}+\frac{\bar{v}}{r} \frac{\partial}{\partial \lambda}\right) \zeta_{s}=0 \quad \forall r \\
& \left(\frac{\partial}{\partial t}+\frac{\bar{v}}{r} \frac{\partial}{\partial \lambda}\right) \zeta_{1}=0 \quad r \neq a .
\end{aligned}
$$

Equation (5.6a) is formally identical to the system solved in section 4, but with the boundary conditions cited above. The corresponding solution in Fourier space is

$$
\hat{\psi}_{\mathrm{s}}(r, t)=\int_{0}^{\infty} G(r, \rho) \hat{\zeta}_{\mathrm{s} 0}(\rho) e^{-\mathrm{i} n \bar{v} t / \rho} \rho \mathrm{d} \rho
$$

where the appropriate Green's function is

$$
G(r, \rho)=-\frac{1}{2 n} \begin{cases}\rho^{-n} r^{n} & 0 \leqslant r \leqslant \rho \\ \rho^{n} r^{-n} & \rho \leqslant r<\infty\end{cases}
$$

and $\hat{\zeta}_{s 0}(\rho)$ is the smooth component of $\hat{\zeta}$ throughout the vortex at time $t=0$.

The Fourier-space equivalent to $(5.6 \mathrm{~b})$ is

$$
\left(\frac{\partial}{\partial t}+\frac{\mathrm{i} n \bar{v}}{r}\right) \hat{\zeta}_{1}=0, \quad r \neq a .
$$

Anticipating that the solution to (5.9) will yield the discrete normal modes which are irrotational on both sides of the mean-state vorticity discontinuity, $\hat{\zeta}_{1}$ is assumed to be separable and of the form

$$
\hat{\zeta}_{1}=\gamma(t) \delta(r-a) \text {. }
$$

Here, $\gamma$ is an undetermined temporal multiplier for $\hat{\zeta}_{1}$, and $\delta(r-a)$ is the Dirac delta function. In terms of the perturbation streamfunction, $(5.10)$ becomes

$$
\nabla^{2} \hat{\psi}_{1}=\gamma(t) \delta(r-a) \text {. }
$$

The streamfunction is also assumed to be separable and of the form $\hat{\psi}_{1}=\gamma(t) \hat{\Psi}_{1}(r)$. Thus, (5.11) becomes

$$
\left(\frac{1}{r} \frac{\mathrm{d}}{\mathrm{d} r}\left(r \frac{\mathrm{d}}{\mathrm{d} r}\right)-\frac{n^{2}}{r^{2}}\right) \hat{\Psi}_{1}=\delta(r-a) .
$$

For $r \neq a,(5.12)$ is Euler's equation. Two conditions are needed to match the solutions in each region across $r=a$. The first is the kinematic boundary condition requiring that the radial velocity $u$ be continuous at $r=a$. Consequently, the Fourier streamfunctionamplitude must be continuous across $r=a$. The second condition results from integrating (5.12) over a small interval that includes $r=a$. This yields the following jump condition for $\hat{\Psi}_{1}$

$$
\frac{\mathrm{d}}{\mathrm{d} r} \hat{\Psi}_{1} r\left(a^{+}\right)-\frac{\mathrm{d}}{\mathrm{d} r} \hat{\Psi}_{1} r\left(a^{-}\right)=1 .
$$

Applying the boundary conditions, and the continuity and jump conditions at $r=a$, yields

$$
\hat{\Psi}_{1}=-\frac{a}{2 n} \begin{cases}a^{-n} r^{n}, & 0 \leqslant r \leqslant a \\ a^{n} r^{-n}, & a \leqslant r<\infty .\end{cases}
$$


To complete the derivation, $\gamma$ must be determined. The remaining constraint is the dynamic boundary condition which requires that the pressure be continuous at $r=a$. In Fourier space, the azimuthal-momentum equation is given by

$$
\frac{\partial \hat{v}}{\partial t}+\frac{i n \bar{v}}{r} \hat{v}+\bar{\eta} \hat{u}=-\frac{i n}{r} \hat{p} .
$$

Evaluating (5.15) on each side of $r=a$ and subtracting gives

$$
\frac{\partial}{\partial t}\left(\hat{v}\left(a^{+}\right)-\hat{v}\left(a^{-}\right)\right)+\frac{\mathrm{i} n}{a}\left(\hat{v}\left(a^{+}\right)-\hat{v}\left(a^{-}\right)\right)-\frac{2}{a} \hat{u}(a)=0 .
$$

In terms of the total streamfunction, equation (5.16) becomes

$$
\frac{\partial}{\partial t}\left(\frac{\partial \hat{\psi}}{\partial r}\left(a^{+}\right)-\frac{\partial \hat{\psi}}{\partial r}\left(a^{-}\right)\right)+\frac{i n}{a}\left(\frac{\partial \hat{\psi}}{\partial r}\left(a^{+}\right)-\frac{\partial \hat{\psi}}{\partial r}\left(a^{-}\right)\right)+\frac{2 \mathrm{i} n}{a^{2}} \hat{\psi}(a)=0 .
$$

Now, from the superposition principle, $\hat{\psi}=\hat{\psi}_{s}+\gamma \hat{\Psi}_{1}$, where $\hat{\psi}_{s}$ and its derivatives are everywhere smooth by construction. Since $\Psi_{1}$ is continuous but has a unit jump in its derivative across $r=a$, equation (5.17) simplifies to

$$
\frac{\mathrm{d} \gamma}{\mathrm{d} t}+\frac{\mathrm{i}}{a}(n-1) \gamma=-\frac{2 \mathrm{i} n}{a^{2}} \hat{\psi}_{s}(a, t)
$$

a first-order linear differential equation for $\gamma$. Upon multiplying through by the integrating factor $\exp \{\mathrm{i}(n-1) t / a\}$ and substituting for $\hat{\psi}_{s}(a, t)$, equation (5.18) becomes

$$
\frac{\mathrm{d}}{\mathrm{d} t}\left(\gamma e^{\mathrm{i}(n-1) r / a}\right)=-\frac{2 \mathrm{i} n}{a^{2}} \int_{0}^{\infty} G(a, \rho) \hat{\zeta}_{s 0} e^{(\mathrm{i}(n-1) / a-\mathrm{i} n \bar{v} / \rho) t} \rho \mathrm{d} \rho .
$$

Integrating in time and then multiplying through by $\exp \{-\mathrm{i}(n-1) t / a\}$ gives

$$
\gamma(t)=-\frac{2 n}{a} \int_{0}^{\infty} \frac{G(a, \rho) \hat{\zeta}_{s 0}}{(n-1-a n \bar{v} / \rho)} e^{-\mathrm{in} \bar{v} t / \rho} \rho \mathrm{d} \rho+c_{1} e^{-\mathrm{i}(n-1) t / a}
$$

where $c_{1}$ is the constant of integration at $t=0$. Equation (5.20) then yields

$$
\begin{aligned}
\gamma(t)= & -\frac{2 n}{a} \int_{0}^{\infty} \frac{G(a, \rho) \hat{\zeta}_{s 0}}{(n-1-a n \bar{v} / \rho)} e^{-\mathrm{i} n \bar{v} t / \rho} \rho \mathrm{d} \rho \\
& +\left(\gamma_{0}+\frac{2 n}{a} \int_{0}^{\infty} \frac{G(a, \rho) \hat{\zeta}_{s 0}}{(n-1-a n \bar{v} / \rho)} \rho \mathrm{d} \rho\right) e^{-\mathrm{i}(n-1) r / a}
\end{aligned}
$$

where $\gamma_{0}$ is the initial amplitude of the normal-mode (Rossby) edge-wave associated with the radial vorticity-gradient of the Rankine swirl. The Fourier streamfunction-amplitude, $\hat{\psi}$, is thus

$$
\begin{aligned}
\hat{\psi}= & \int_{0}^{\infty} G(r, \rho) \hat{\zeta}_{s 0} e^{-\mathrm{i} n \bar{v} t / \rho} \rho \mathrm{d} \rho \\
& -\frac{2 n}{a} \hat{\Psi}_{1} \int_{0}^{\infty} \frac{G(a, \rho) \hat{\zeta}_{s 0}}{(n-1-a n \bar{v} / \rho)} e^{-\mathrm{i} n \bar{v} t / \rho} \rho \mathrm{d} \rho \\
& +\hat{\Psi}_{1}\left(\gamma_{0}+\frac{2 n}{a} \int_{0}^{\infty} \frac{G(a, \rho) \hat{\zeta}_{s 0}}{(n-1-a n \bar{v} / \rho)} \rho \mathrm{d} \rho\right) e^{-\mathrm{i}(n-1) t / a}
\end{aligned}
$$


where $G$ is given by (5.8) and $\hat{\Psi}_{1}$ is given by (5.14). To obtain the physical-space streamfunction, the inverse Fourier transform must be applied to (5.22).

Equation (5.22) may be given a simple interpretation. The first line may be identified with the continuous-spectrum solution and represents the unbounded analogue of the solution presented in section 4 . The second line is a conversion term that transfers a portion of the KE from the continuous-spectrum solution into the discrete mode (third of the three terms on the right-hand side of (5.22)). Note that even with no normal-mode component initially, i.e. $\gamma_{0}=0$, the continuous-spectrum solution always projects onto the normal mode at later times provided the integral does not vanish (see Fig. 18; cf. Farrell 1984). For $n \neq 1$, the discrete modes rotate more slowly than the vortex and represent retrogressing Rossby edge-waves at $r=a+\epsilon$. It may be shown that, in the absence of perturbation vorticity inside the radius of maximum winds, as $t \rightarrow \infty$ only the edge-wave component remains. In particular, the asymptotic solution for $n=1$ is the non-rotating normal-mode associated with a translation of the basic-state vortex. On the other hand, for non-zero perturbation vorticity inside the radius of maximum winds, the asymptotic solution has both a normal-mode and continuous-spectrum response. In this case, the solution for $n=1$ has an oscillatory component. In hurricanes, where the basic-state angular velocity has a small but generally non-zero radial gradient inside the radius of maximum winds, as $t \rightarrow \infty$ the latter effect would probably be of little significance. At early times, however, the transient wave-number-one component could produce a cycloidal track in the fully non-linear formulation.
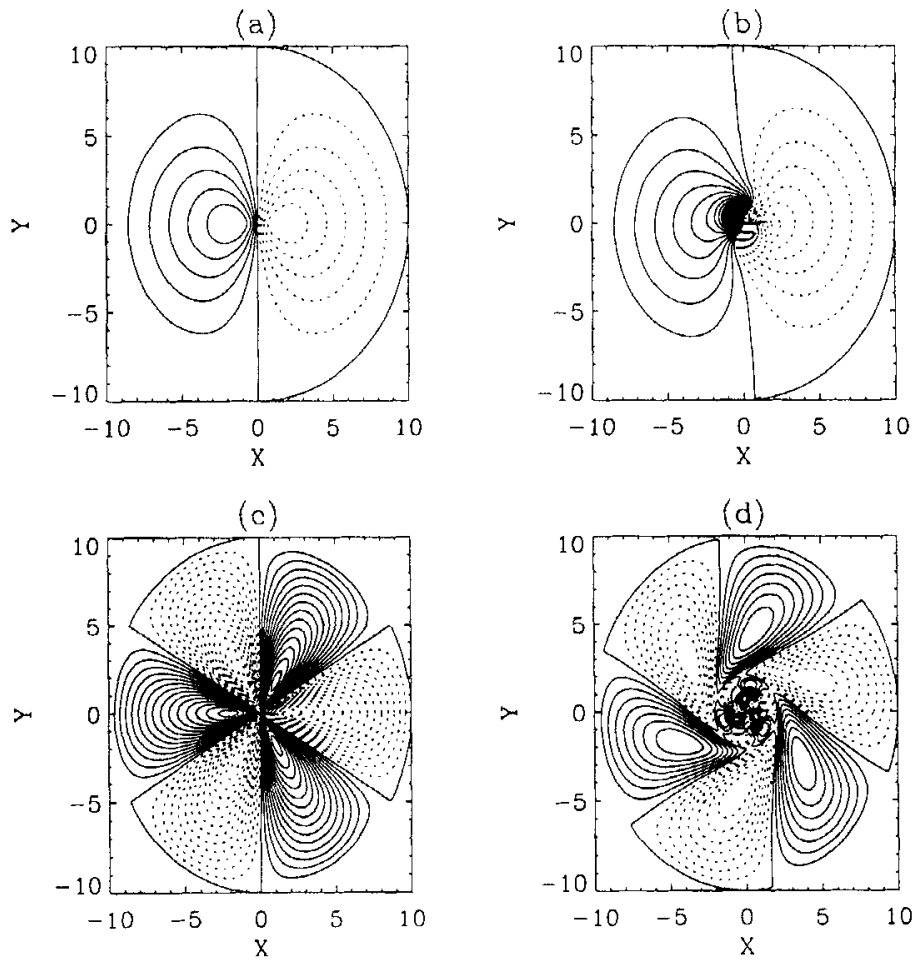

Figure 18. Perturbation streamfunction forced by initial vorticity-profile $\hat{\zeta}_{0}=1 /(r+1)^{3}$. Panels (a) and (b) are for wave number $n=1$ and the contour interval is $1.59 \times 10^{-2}$. Panels (c) and (d) are for $n=3$ and the contour interval is $1.06 \times 10^{-3}$. The columns are for times $t=0.0$ and $t=7.2$ respectively. Solid lines denote $\psi \geqslant 0$; dotted lines denote $\psi<0$. 
The above model has been extended to an arbitrary number of uniform-vorticity regions (Edwards 1994). Sutyrin (1989) developed the quasi-geostrophic shallow-water equivalent of the multi-region model. However, his formulation did not explicitly describe the interaction between the continuous- and discrete-spectrum solutions. Extension of the above model to a quasi-geostrophic and $\mathrm{AB}$ shallow-water model would be of interest since it could prove useful in theoretical studies of hurricane track.

\section{DisCUSSION AND CONCLUSION}

The inviscid dynamics of sheared disturbances has been investigated within the framework of non-divergent vorticity dynamics. Particular emphasis has been placed on understanding the wave-number and spatial structure dependencies in perturbation decay.

A truly inviscid mechanism which favours the decay of high-wave-number perturbations over low-wave-number perturbations was identified. In particular, wave-number-one disturbances decayed more slowly than all others. In the development of AB theory, SM appealed to both observational and computational evidence of a wave-number-selection mechanism as the basis for the theory. The analytical results presented here elucidate an important wave-number-selection mechanism and provide further theoretical support for the validity of $\mathrm{AB}$ theory. Although diffusion acting over sufficiently long times preferentially damps the high wave-numbers over the low wave-numbers, this paper reveals an underlying dynamic that operates at early times and greatly accelerates this preferential decay.

The spatial structure of the initial conditions has been shown to play a significant role in disturbance evolution. The spatial scale controlled the rates at which a disturbance sheared and subsequently decayed. In the swirling model, the radial location of the initial condition was important since the shear decreased rapidly outside the radius of maximum winds. These factors led to the definition of an effective shear which accounted for the interaction of the symmetric basic-state and the initial vorticity-profile. As defined here, the effective shear was shown to be an adequate estimate for the higher-wave-number asymmetries, provided that the radial decrease of the initial condition was greater than that of the basic-state angular velocity. However, the effective shear was a significant underestimate for the lower wave-numbers.

Using the effective shear, decay half-lives were estimated on a wave number by wave number basis. The results clearly demonstrated that the half-lives do not decrease as $n^{-2}$. This refutes the CW suggestion that the damping rate is proportional to the square of the AWN. The effective half-lives for wave number one were found to be approximately seven times those calculated using the effective shear. This was used to estimate half-lives for tangential-wind profiles that are more representative of hurricanes outside their radius of maximum winds. These half-lives appear to be reasonable estimates for the simulated asymmetric forcings.

The wind profiles considered did not have a basic-state vorticity-gradient and, thus, excluded discrete normal-modes from the solution set. An analytical method capturing both continuous- and discrete-solution components valid for all AWNs, was presented in section 5 where the Rankine vortex was extended into a uniformly rotating core. The interaction of the continuous spectrum and the discrete normal modes has been extensively studied for simple rectilinear models (e.g. Pedlosky 1964 and Farrell 1984), but a thorough investigation into swirling flows remains for future work.

Since the basic-state vorticity-gradient in a tropical cyclone or polar vortex may be viewed as an effective vortex $\beta$, CW suggested that this effective $\beta$ would introduce a retrogression that could counteract the rate of perturbation tilting induced by the differential 
rotation. Disturbance vorticity would then no longer be conserved following the basic-state swirl and initially 'upright' asymmetries could also experience a transient growth prior to their ultimate decay. A thorough examination of such growth processes and other related behaviour, using idealized evolutionary models should prove useful in the interpretation of observations describing interactions between tropical cyclones and migratory disturbances in the storm's environment (Molinari 1992). These ideas will be explored in a future paper.

The importance of vortex axisymmetrization in theories of two-dimensional turbulence has been noted previously by Moffat (1986) and Sutyrin (1989). Sutyrin characterized the transfer of energy from the azimuthal perturbations to the circular basic-state as being analogous to the inverse energy-cascade to large scales, while the growth of the disturbance PV gradient was associated with the transfer of enstrophy to small scales. Further investigation and quantification of these ideas in real tropical cyclones would be of great dynamical interest.

\section{ACKNOWLEDGEMENTS}

This research was partially supported by the Office of Naval Research (grant number N00014-93-1-0456) and the National Science Foundation (grant number ATM-9312655). Mr. Smith was supported through the Air Force Institute of Technology program. The authors thank Mr. Randall Kallenbach for proofreading the manuscript.

\section{APPENDIX}

The verification of the numerical model is summarized here. Since the meridional, or radial, scale of the disturbance generally changes with time, we need to estimate when the disturbance scale becomes smaller than the resolution of the model. In the rectilinear model of section 2 the meridional wave-number of an upright-plane-wave perturbation is found to be $l=-k S t$. Thus, a meridional wavelength is given by $2 \pi / k S t$. Assuming the model resolves perturbations that have scales greater than or equal to twice the grid spacing, the model no longer resolves the perturbations when

$$
\frac{2 \pi}{k S t} \leqslant 2 \Delta y
$$

where $\Delta y$ is the grid spacing, $k$ is the zonal wavenumber, and $S$ is the shear. Thus,

$$
S t \geqslant \frac{\pi}{k \Delta y}
$$

gives an estimate of when the perturbations are no longer resolvable. Whilst (A.2) is exact for infinite plane-wave disturbances, it more generally represents an upper time-limit for initial conditions for vorticity of finite meridional extent. In such cases a lower time-limit can also be deduced. For initial meridional scales that are large compared with the grid spacing, however, the two estimates are identical to leading order. In sections 2 and 3 , the worst model resolution was $\Delta y=0.056$, corresponding to 361 meridional grid points and integration limits at \pm 10 . For $k=3$, the model resolution starts becoming inadequate for $S t \approx 20$.

For the swirling model, SM have shown that the change in the radial wave-number with time for a tightly wound asymmetry is

$$
\frac{\mathrm{d} k}{\mathrm{~d} t}=-n \frac{\mathrm{d} \bar{\Omega}}{\mathrm{d} r} .
$$


Here, $k$ is the local radial wave-number, $n$ is the azimuthal wave-number, and $\bar{\Omega}$ is the basic-state angular velocity. For the Rankine profile (5.1), (A.3) is

$$
\frac{\mathrm{d} k}{\mathrm{~d} t}=-\frac{2 n}{r^{3}} .
$$

Although not strictly valid for an initially upright asymmetry, (A.4) may nonetheless be used to estimate when the resolution in the swirling model becomes inadequate. Integrating (A.4) for an initially upright asymmetry yields

$$
k=-\frac{2 n t}{r^{3}}
$$

For a radial grid spacing $\Delta r$, the model resolution starts becoming inadequate when

$$
\frac{2 \pi}{k} \leqslant 2 \Delta r
$$

or, when

$$
t \approx \frac{\pi r^{3}}{2 n \Delta r} .
$$

In section 4 , the lowest model-resolution was $\Delta r=0.05$, corresponding to boundaries $a=1$ and $b=10$ with 181 grid points. Thus, at the radius of maximum winds for $n=3$, (A.7) predicts that the asymmetries start becoming smaller than the model resolution when $t \approx 10$. However, at $r=5$ and $n=3$, the asymmetries start becoming unresolvable when $t \approx 1300$. In the foregoing discussion, the effect of an initially finite radial vorticity scale on the resolution time limit $\left(t_{\text {MAX }}\right)$ was neglected. Although finite disturbance scales slightly decrease $t_{\mathrm{MAX}}$ in the rectilinear model, the radial dependence of the azimuthal scale in the swirl model can result in a substantially smaller $t_{\text {MAX }}$ than the estimate (A.7). This discrepancy is most acute in the near-vortex region where meridians begin to converge. Since the bounded Rankine flow of section 4 did not include $r=0$ the predictions of (A.7) were found to be adequate for the radial resolutions chosen.

To be consistent with the analytical system, the model results must satisfy the momentum equations to within discretization error. Since the trapezoidal rule is used in this paper, the discretization error should be proportional to the square of model resolution. The procedure used to evaluate the output of the swirling model is discussed below.

For the bounded Rankine vortex (4.7), model verification was performed with the Fourier-space representation of the momentum equations

$$
\begin{aligned}
& \frac{\partial \hat{u}}{\partial t}+\frac{\mathrm{i} n}{r^{2}} \hat{u}-\bar{\xi} \hat{v}=-\frac{\partial \hat{p}}{\partial r} \\
& \frac{\partial \hat{v}}{\partial t}+\frac{\mathrm{i} n}{r^{2}} \hat{v}+\bar{\eta} \hat{u}=-\frac{\mathrm{i} n}{r} \hat{p},
\end{aligned}
$$

where $\bar{\xi}=f+2 \bar{v} / r$ is the modified Coriolis parameter and other notation is as defined in the main text.

From (A.8b), $\hat{p}$ was found for all radial values at a fixed time. Using centreddifference approximations for all differentiated quantities, the radial gradient of $\hat{p}$ was calculated and compared to the left side of (A.8a). For the shear-induced asymmetry from $\mathrm{CW}\left(\hat{\zeta}_{0}=-i / r^{2}, n=2\right)$ and model resolutions of $\Delta r=\Delta t=5 \times 10^{-2}$ at $t=9$, the difference between the left and right sides of (A.8a) at $r=2.3$ was $-2.54 \times 10^{-5}$. For 
double and quadruple the above model resolution, the differences were $-6.34 \times 10^{-6}$ and $-1.59 \times 10^{-6}$ respectively. In each case, as the model resolution doubled, the difference decreased by a factor of four. For the same model resolutions at $r=1.7$ and $t=5.9$, the differences were $9.02 \times 10^{-6}, 2.01 \times 10^{-6}$ and $4.87 \times 10^{-7}$ respectively. The model output was checked for a variety of times, radial locations and initial vorticity-profiles. In all cases, model output was found to be quadratically convergent.

\section{REFERENCES}

Carr, L. E. III and Williams, R. T. 1989

Case, K. M.

Dritschell, D. C.

Edwards, J. P.

Eliassen, A.

Farrell, B.

Guinn, T. A. and Schubert, W. H.

Lamb, $\mathrm{H}$.

Lindzen, R. S.

McCalpin, J. D.

Melander, M. V., McWilliams, J. C. and Zabusky, N. J.

Moffat, H. K.

Molinari, J.

Montgomery, M. T. and Farrell, B. F.

\section{Orr, W. McF.}

Pedlosky, J.

Pullin, D. I.

Pullin, D. I., Jacobs, P. A., Grimshaw, R. H. J. and Saffman, P. G.

Saffman, P. G.

Shapiro, L. J.

Shapiro, L. J. and Montgomery, $\mathrm{M}$. T.

Shapiro, L. J. and Ooyama, K. V.

Smith, R. K. and Weber, H. C.
1960

1988

1994

1951

1982

1984

1987

1993

1932

1990

1987

1987

1986

1992

1990

1991

1907

1964

1981

1989

1992

1992

1993

1990

1993

Barotropic vortex stability to perturbations from axisymmetry. $J$. Atmos. Sci., 46, 3177-3191

Stability of inviscid plane Couette flow. Phys. Fluids, 3, 143-148

The repeated filamentation of two-dimensional vorticity interfaces. J. Fluid Mech., 194, 511-547

'Barotropic instability in the inner core of tropical cyclones'. Atmospheric Science Paper No. 552, Colorado State University, Fort Collins, $\mathrm{CO}$

Slow thermally or frictionally controlled meridional circulation in a circular vortex. Astrophys. Norv., 5, 19-60

The initial growth of disturbances in a baroclinic flow. J. Atmos. Sci., 39, 1663-1686

Modal and non-modal baroclinic waves. J. Atmos. Sci., 41, 668673

Developing disturbances in shear. J. Atmos. Sci., 40, 2191-2199

Hurricane spiral bands. J. Atmos. Sci., 50, 3380-3403

Hydrodynamics. Dover Publications

Dynamics in Atmospheric Physics. Cambridge University Press

On the adjustment of azimuthally perturbed vortices. $J$. Geophys. Res., 92, 8213-8225

Axisymmetrization and vorticity-gradient intensification of an isolated two-dimensional vortex through filamentation. J. Fluid Mech., 178, 137-159

'Geophysical and astrophysical turbulence'. Pp. 228-244 in Advances in Turbulence, G. Comte-Ballot and J. Mathieu, eds., Springer Verlag

'Environmental controls on eye wall cycles and intensity change in Hurricane Allen (1980)'. Pp. 328-337 in ICSU/WMO International Symposium on Tropical Cyclone Disasters, October 12-16, 1992, Beijing

Dry surface frontogenesis arising from interior potential vorticity perturbations in a semigeostrophic model. J. Atmos. Sci., 47, 2837-2852

Moist surface frontogenesis associated with interior potential vorticity anomalies in a semi-geostrophic model. J. Atmos. Sci., 48, 343-367

Stability or instability of the steady motions of a perfect fluid. Proc. Roy. Irish Acad., A29, 9-68

An initial-value problem in the theory of baroclinic instability. Tellus, 16, 12-17

The nonlinear behavior of a constant vorticity layer at a wall. $J$. Fluid Mech., 108, 401-421

Instability and filamentation of finite-amplitude waves on vortex layers of finite thickness. J. Fluid Mech., 209, 359-384

Vortex Dynamics. Cambridge University Press

Hurricane vortex motion and evolution in a three-layer model. $J$. Atmos. Sci. , 49, 140-153

A three-dimensional balance theory for rapidly rotating vortices. J. Atmos. Sci., 50, 3322-3335

Barotropic vortex evolution on a beta plane. J. Atmos. Sci., 47, $170-187$

An extended analytic theory of tropical-cyclone motion in a barotropic shear flow. Q. J. R. Meteorol. Soc., 119, 1149 1166 
Sutyrin, G. G.

Thomson, W.

Willoughby, H. E.
1989 Azimuthal waves and symmetrization of an intense vortex. Sov. Phys. Dokl., 34, 104-106

1880 On the vibrations of a columnar vortex. Phil. Mag., 5, 155-168 1887 Stability of Fluid Motion: Rectilinear Motion of Viscous Fluid Between Two Parallel Planes. Phil. Mag., 24, 188-196

1977 Inertia-buoyancy waves in hurricanes. J. Atmos. Sci., 34, 10281039

1979 Excitation of spiral bands in hurricanes by interaction between the symmetric mean vortex and a shearing environmental steering current. J. Atmos. Sci., 36, 1226-1235

1988 Linear motion of a shallow-water, barotropic vortex. J. Atmos. Sci. 45, 1906-1928 